\title{
Dynamic Type Inference for Gradual Hindley-Milner Typing
}

\author{
YUSUKE MIYAZAKI*, Kyoto University, Japan \\ TARO SEKIYAMA, National Institute of Informatics, Japan \\ ATSUSHI IGARASHI, Kyoto University, Japan
}

Garcia and Cimini study a type inference problem for the ITGL, an implicitly and gradually typed language with let-polymorphism, and develop a sound and complete inference algorithm for it. Soundness and completeness mean that, if the algorithm succeeds, the input term can be translated to a well-typed term of an explicitly typed blame calculus by cast insertion and vice versa. However, in general, there are many possible translations depending on how type variables that were left undecided by static type inference are instantiated with concrete static types. Worse, the translated terms may behave differently-some evaluate to values but others raise blame.

In this paper, we propose and formalize a new blame calculus $\lambda_{\mathrm{B}}^{\mathrm{DTI}}$ that avoids such divergence as an intermediate language for the ITGL. A main idea is to allow a term to contain type variables (that have not been instantiated during static type inference) and defer instantiation of these type variables to run time. We introduce dynamic type inference (DTI) into the semantics of $\lambda_{\mathrm{B}}^{\mathrm{DTI}}$ so that type variables are instantiated along reduction. The DTI-based semantics not only avoids the divergence described above but also is sound and complete with respect to the semantics of fully instantiated terms in the following sense: if the evaluation of a term succeeds (i.e., terminates with a value) in the DTI-based semantics, then there is a fully instantiated version of the term that also succeeds in the explicitly typed blame calculus and vice versa.

Finally, we prove the gradual guarantee, which is an important correctness criterion of a gradually typed language, for the ITGL.

CCS Concepts: • Theory of computation $\rightarrow$ Operational semantics; $\bullet$ Software and its engineering $\rightarrow$ Functional languages; Dynamic analysis; Polymorphism;

Additional Key Words and Phrases: gradual typing, dynamic type inference, gradual guarantee

ACM Reference Format:

Yusuke Miyazaki, Taro Sekiyama, and Atsushi Igarashi. 2019. Dynamic Type Inference for Gradual HindleyMilner Typing. Proc. ACM Program. Lang. 3, POPL, Article 18 (January 2019), 29 pages. https://doi.org/10.1145/ 3290331

\section{INTRODUCTION}

\subsection{Gradual Typing}

Statically and dynamically typed languages have complementary strengths. On the one hand, static typing provides early detection of bugs, but the enforced programming style can be too constrained, especially when the type system is not very expressive. On the other hand, dynamic typing is better

${ }^{*}$ Current affiliation: Recruit Co., Ltd.

Authors' addresses: Yusuke Miyazaki, Graduate School of Informatics, Kyoto University, Kyoto, Japan, miyazaki@fos kuis.kyoto-u.ac.jp; Taro Sekiyama, National Institute of Informatics, Tokyo, Japan, tsekiyama@acm.org; Atsushi Igarashi, Graduate School of Informatics, Kyoto University, Kyoto, Japan, igarashi@kuis.kyoto-u.ac.jp.

This work is licensed under a Creative Commons Attribution 4.0 International License.

(c) 2019 Copyright held by the owner/author(s).

2475-1421/2019/1-ART18

https://doi.org/10.1145/3290331

Proc. ACM Program. Lang., Vol. 3, No. POPL, Article 18. Publication date: January 2019. 
suited for rapid prototyping and fast adaption to changing requirements, but error detection is deferred to run time.

There has been much work to integrate static and dynamic typing in a single programming language [Abadi et al. 1991; Bracha and Griswold 1993; Cartwright and Fagan 1991; Flanagan and Felleisen 1999; Siek and Taha 2006; Thatte 1990; Tobin-Hochstadt and Felleisen 2008]. Gradual typing [Siek and Taha 2006] is a framework to enable seamless code evolution from a fully dynamically typed program to a fully statically typed one within a single language. The notion of gradual typing has been applied to various language features such as objects [Siek and Taha 2007], generics [Ina and Igarashi 2011], effects [Bañados Schwerter et al. 2014, 2016], ownership [Sergey and Clarke 2012], parametric polymorphism [Ahmed et al. 2011; Igarashi et al. 2017; Xie et al. 2018] and so on. More recently, even methodologies to "gradualize" existing statically typed languages systematically, i.e., to generate gradually typed languages, are also studied [Cimini and Siek 2016, 2017; Garcia et al. 2016].

The key notions in gradual typing are the dynamic type and type consistency. The dynamic type, denoted by $\star$, is the type for the dynamically typed code. For instance, a function that accepts an argument of type $\star$ can use it in any way and the function can be applied to any value. So, both $(\lambda x: \star \cdot x+2) 3$ and $(\lambda x: \star \cdot x+2)$ true are well-typed programs in a gradually typed language. To formalize such loose static typechecking, a type consistency relation, denoted by $\sim$, on types replaces some use of type equality. In the typing rule for applications, the function argument type and the type of an actual argument are required not to be equal but to be consistent; also, both $\star \sim$ int and $\star \sim$ bool hold, making the two terms above well typed.

The semantics of a gradually typed language is usually defined by a "cast-inserting" translation into an intermediate language with explicit casts, which perform run-time typechecking. For example, the two examples above can be translated into terms of the blame calculus [Wadler and Findler 2009] as follows.

$$
\begin{aligned}
(\lambda x: \star \cdot x+2) 3 & \rightsquigarrow\left(\lambda x: \star \cdot\left(x: \star \Rightarrow^{\ell_{1}} \text { int }\right)+2\right)\left(3: \text { int } \Rightarrow^{\ell_{2}} \star\right) \\
(\lambda x: \star \cdot x+2) \text { true } & \rightsquigarrow\left(\lambda x: \star \cdot\left(x: \star \Rightarrow^{\ell_{1}} \text { int }\right)+2\right)\left(\text { true }: \text { bool } \Rightarrow^{\ell_{2}} \star\right)
\end{aligned}
$$

Here, $\rightsquigarrow$ denotes cast-inserting translation. The term of the form $f: U_{1} \Rightarrow^{\ell} U_{2}$ is a cast of $f$ from type $U_{1}$ to $U_{2}$ and appears where typechecking was loosened due to type consistency. ${ }^{1}$ In these examples, actual arguments 3 and true are cast to $\star$ and $x$ of type $\star$ is cast to int before being passed to + , which expects an integer. In what follows, a sequence of casts $\left(f: U_{1} \Rightarrow^{\ell_{1}} U_{2}\right): U_{2} \Rightarrow{ }^{\ell_{2}} U_{3}$ is often abbreviated to $f: U_{1} \Rightarrow^{\ell_{1}} U_{2} \Rightarrow^{\ell_{2}} U_{3}$.

The former term evaluates to 5 whereas the latter to an uncatchable exception, called blame [Findler and Felleisen 2002], blame $\ell_{1}$, indicating that the cast on $x$ fails:

$$
\begin{aligned}
\left(\lambda x: \star .\left(x: \star \Rightarrow^{\ell_{1}} \text { int }\right)+2\right)\left(3: \text { int } \Rightarrow^{\ell_{2}} \star\right) & \longmapsto\left(3: \text { int } \Rightarrow^{\ell_{2}} \star^{\ell_{1}} \text { int }\right)+2 \\
& \longmapsto 3+2 \\
& \longmapsto 5 \\
\left(\lambda x: \star .\left(x: \star \Rightarrow^{\ell_{1}} \text { int }\right)+2\right)\left(\text { true }: \text { bool } \Rightarrow^{\ell_{2}} \star\right) & \left.\longmapsto \text { (true : bool } \Rightarrow^{\ell_{2}} \star^{\ell_{1}} \text { int }\right)+2 \\
& \longmapsto \text { blame } \ell_{1}+2 \\
& \longmapsto \text { blame } \ell_{1}
\end{aligned}
$$

The terms 3 : int $\Rightarrow^{\ell_{2}} \star$ and true : bool $\Rightarrow^{\ell_{2}} \star$ are values in the blame calculus and they can be understood as an integer and Boolean, respectively, tagged with its type. Being values, they are passed to the function as they are. The cast from $\star$ to int investigates if the tag of the target is int;

\footnotetext{
${ }^{1}$ The symbol $\ell$ is called a blame label and is used to identify a cast. Following Siek et al. [2015a], we use $f$ for the intermediate language and save $e$ for the surface language ITGL.
} 
if it is, the tag is removed and the untagged integer is passed to +; otherwise, blame is raised with information on which cast has failed.

\subsection{Type Inference for Gradual Typing}

Type inference (a.k.a. type reconstruction) for languages with the dynamic type has been studied. Siek and Vachharajani [2008] proposed a unification-based type inference algorithm for a gradually typed language with simple types. Garcia and Cimini [2015] later proposed a type inference algorithm with a principal type property for the Implicitly Typed Gradual Language (ITGL) with and without let-polymorphism. More recently, Xie et al. [2018] studied an extension of the OderskyLäufer type system for higher-rank polymorphism [Odersky and Läufer 1996] with the dynamic type and bidirectional algorithmic typing for it. Also, Henglein and Rehof [1995] studied a very close problem of translation from an untyped functional language to an ML-like language with coercions [Henglein 1994], using a constraint-based type inference.

The key idea in Garcia and Cimini's work (inherited by Xie et al.) is to infer only static types-that is, types not containing $\star-$ for where type annotations are omitted. For example, for

$$
(\lambda x . x 2)(\lambda y \text { :int. } y) \text {, }
$$

the type inference algorithm outputs the following fully annotated term:

$$
e=(\lambda x: \text { int } \rightarrow \text { int. } x 2)(\lambda y: \text { int. } y) \text {. }
$$

The idea of inferring only static types is significant for the principal type property because it excludes terms like $(\lambda x: \star \rightarrow$ int. $x 2)(\lambda y:$ int. $y),(\lambda x:$ int $\rightarrow \star . x 2)(\lambda y:$ int. $y)$, and $(\lambda x: \star . x 2)(\lambda y:$ int. $y)$, which are all well typed in the gradual type system but not obtained by applying type substitution to $e$. Based on this idea, they showed that the ITGL enjoys the principal type property, which means that if there are type annotations to make a given term well typed, the type inference succeeds and its output subsumes all other type annotations that make it well typed-in the sense that they are obtained by applying some type substitution.

\subsection{Incoherence Problem}

Unlike ordinary typed $\lambda$-calculi, however, the behavior of a term depends on concrete types chosen for missing type annotations. For example, for the following term

$$
(\lambda x: \star . x 2)(\lambda y \cdot y)
$$

it is appropriate to recover any static type $T$ for $y$ if we are interested only in obtaining a well-typed term because $\star \sim T \rightarrow T$ but the evaluation of the resulting term significantly differs depending on the choice for $T$. To see this, let's translate $(\lambda x: \star . x 2)(\lambda y: T . y)$ (of type $\star$ ). It is translated to

$$
\left(\lambda x: \star .\left(x: \star \Rightarrow^{\ell_{1}} \star \rightarrow \star\right)\left(2: \text { int } \Rightarrow^{\ell_{2}} \star\right)\right)\left((\lambda y: T \cdot y): T \rightarrow T \Rightarrow^{\ell_{3}} \star\right)
$$

regardless of $T$. If $T=$ int, then it reduces to value $2:$ int $\Rightarrow^{\ell_{3}} \star$ as follows:

$$
\begin{aligned}
& \left(\lambda x: \star .\left(x: \star \Rightarrow^{\ell_{1}} \star \rightarrow \star\right)\left(2: \text { int } \Rightarrow^{\ell_{2}} \star\right)\right)\left((\lambda y: \text { int. } y): \text { int } \rightarrow \text { int } \Rightarrow^{\ell_{3}} \star\right) \\
\longmapsto & \left((\lambda y: \text { int. } y)\left(2: \text { int } \Rightarrow^{\ell_{2}} \star \Rightarrow^{\bar{\ell}_{3}} \text { int }\right)\right): \text { int } \Rightarrow^{\ell_{3}} \star \\
\longmapsto \quad & ((\lambda y: \text { int. } y) 2): \text { int } \Rightarrow^{\ell_{3}} \star \\
& 2: \text { int } \Rightarrow^{\ell_{3}} \star
\end{aligned}
$$

but, if $T=$ bool, it reduces to blame $\bar{\ell}_{3}$ as follows:

$$
\begin{aligned}
&\left(\lambda x: \star .\left(x: \star \Rightarrow^{\ell_{1}} \star \rightarrow \star\right)\left(2: \text { int } \Rightarrow^{\ell_{2}} \star\right)\right)\left((\lambda y: \text { bool. } y): \text { bool } \rightarrow \text { bool } \Rightarrow^{\ell_{3}} \star\right) \\
& \longmapsto^{*} \quad\left((\lambda y: \text { bool. } y)\left(2: \text { int } \Rightarrow^{\ell_{2}} \star \Rightarrow^{\bar{\ell}_{3}} \text { bool }\right)\right): \text { bool } \Rightarrow^{\ell_{3}} \star \\
& \longmapsto \quad \text { blame } \bar{\ell}_{3}
\end{aligned}
$$


(the shaded subterm is the source of blame and the blame label $\bar{\ell}_{3}$, which is the negation of $\ell_{3}$, means that a functional cast labeled $\ell_{3}$ has failed due to type mismatch on the argument, not on the return value). Xie et al. [2018] face the same problem in a slightly different setting of a higher-rank polymorphic type system with the dynamic type and point out that their type system is not coherent [Breazu-Tannen et al. 1991] in the sense that the run-time behavior of the same source program depends on the particular choice of types.

Garcia and Cimini [2015] do not clearly discuss how to deal with this problem. Given term $(\lambda x: \star . x 2)(\lambda y \cdot y)$, their type reconstruction algorithm outputs $(\lambda x: \star . x 2)(\lambda y: Y \cdot y)$, where $Y$ is a type variable that is left undecided and they suggest those undecided variables be replaced by type parameters but their semantics is left unclear. One possibility would be to understand type parameters as distinguished base types (without constants) but it would also make the execution fail because int $\neq Y$. The problem here is that the only choice for $T$ that makes execution successful is int but it is hard to see statically.

An alternative, which is close to what Henglein and Rehof [1995] do and also what Xie et al. [2018] suggest, is to substitute the dynamic type $\star$ for these undecided type variables. If we replace $Y$ with $\star$ in the example above, we will get

$$
\begin{aligned}
& \left(\lambda x: \star .\left(x: \star \Rightarrow^{\ell_{1}} \star \rightarrow \star\right)\left(2: \text { int } \Rightarrow^{\ell_{2}} \star\right)\right)\left((\lambda y: \star \cdot y): \star \rightarrow \star \Rightarrow^{\ell_{3}} \star\right) \\
& \longmapsto^{*} \quad(\lambda y: \star \cdot y)\left(2: \text { int } \Rightarrow^{\ell_{2}} \star\right) \\
& \longmapsto \quad 2: \text { int } \Rightarrow^{\ell_{2}} \star \text {. }
\end{aligned}
$$

As far as this example is concerned, substitution of $\star$ sounds like a good idea as, if there is static-type substitution that makes execution successful (i.e., terminate at a value), substitution of $\star$ is expected to make execution also successful-this is part of the gradual guarantee property [Siek et al. 2015a].

However, substitution of $\star$ can make execution successful, even when there is no static type that makes execution successful. For example, let's consider the ITGL term

$$
(\lambda x: \star \rightarrow \star \rightarrow \star . x 2 \text { true })\left(\lambda y_{1} \cdot \lambda y_{2} \text {. if } b \text { then } y_{1} \text { else } y_{2}\right)
$$

where if $e_{1}$ then $e_{2}$ else $e_{3}$ requires $e_{2}$ and $e_{3}$ to have the same type and $b$ is a (statically typed) Boolean term that refers to neither $y_{1}$ nor $y_{2}$. For this term, the type inference algorithm outputs $(\lambda x: \star \rightarrow \star \rightarrow \star . x 2$ true $)\left(\lambda y_{1}: Y . \lambda y_{2}: Y\right.$. if $b$ then $y_{1}$ else $\left.y_{2}\right)$ and, if we substitute $\star$ for $Y$, then it executes as follows:

$(\lambda x: \star \rightarrow \star \rightarrow \star$. $x 2$ true $)\left(\lambda y_{1}: \star . \lambda y_{2}: \star\right.$. if $b$ then $y_{1}$ else $\left.y_{2}\right)$

$\rightsquigarrow \quad\left(\lambda x: \star \rightarrow \star \rightarrow \star\right.$. $x\left(2:\right.$ int $\left.\Rightarrow^{\ell_{1}} \star\right)\left(\right.$ true : bool $\left.\left.\Rightarrow^{\ell_{2}} \star\right)\right)\left(\lambda y_{1}: \star . \lambda y_{2}: \star\right.$. if $b$ then $y_{1}$ else $\left.y_{2}\right)$

$\longmapsto^{*} \quad$ if $b$ then $\left(2:\right.$ int $\left.\Rightarrow^{\ell_{1}} \star\right)$ else (true : bool $\left.\Rightarrow^{\ell_{2}} \star\right)$

$\longmapsto^{*} \begin{cases}2: \text { int } \Rightarrow^{\ell_{1}} \star & \left(\text { if } b \longmapsto^{*} \text { true) }\right. \\ \text { true : bool } \Rightarrow^{\ell_{2}} \star & (\text { if } b \longmapsto * \text { false) }\end{cases}$

but, if we substitute a static type $T$ for $Y$, then it results in blame due to a failure of either of the shaded casts:

$$
\begin{aligned}
& (\lambda x: \star \rightarrow \star \rightarrow \star . x 2 \text { true })\left(\lambda y_{1}: T . \lambda y_{2}: T \text {. if } b \text { then } y_{1} \text { else } y_{2}\right) \\
& \rightsquigarrow \quad\left(\lambda x: \star \rightarrow \star \rightarrow \star \text {. } x\left(2: \text { int } \Rightarrow^{\ell_{1}} \star\right)\left(\text { true : bool } \Rightarrow^{\ell_{2}} \star\right)\right) \\
& \left(\left(\lambda y_{1}: T . \lambda y_{2}: T \text {. if } b \text { then } y_{1} \text { else } y_{2}\right): T \rightarrow T \rightarrow T \Rightarrow^{\ell_{3}} \star \rightarrow \star \rightarrow \star\right) \\
& \longmapsto^{*} \quad\left(\left(\left(\lambda y_{1}: T \text {. } \lambda y_{2}: T \text {. if } b \text { then } y_{1} \text { else } y_{2}\right)\left(2: \text { int } \Rightarrow^{\ell_{1}} \star \Rightarrow^{\bar{\ell}_{3}} T\right)\right): T \rightarrow T \Rightarrow^{\ell_{3}} \star \rightarrow \star\right) \\
& \text { (true : bool } \Rightarrow^{\ell_{2}} \star \text { ) }
\end{aligned}
$$

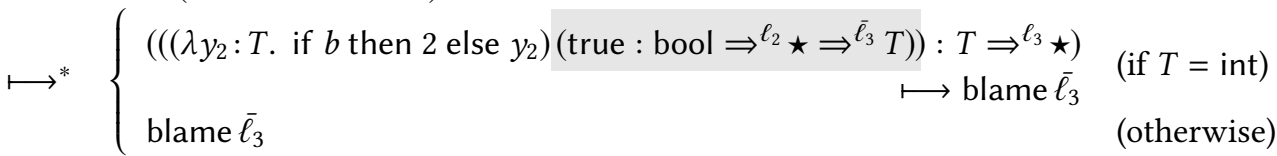


Substitution of $\star$ is not only against the main idea that only static types are inferred for missing type annotations but also not very desirable from the viewpoint of program evolution and early bug detection: Substitution of $\star$ inadvertently hides the fact that there is no longer a hope for a given term (in this case $\left(\lambda x: \star \rightarrow \star \rightarrow \star . x 2\right.$ true) $\left(\lambda y_{1} . \lambda y_{2}\right.$. if $b$ then $y_{1}$ else $\left.\left.y_{2}\right)\right)$ to be able to evolve to $a$ well-typed term by replacing occurrences of $\star$ with static types. This concealment of potential errors would be disappointing for languages where it is hoped to detect as early as possible programs that the underlying static type system does not accept after evolution.

\subsection{Our Work: Dynamic Type Inference}

In this work, we propose and formalize a new blame calculus $\lambda_{\mathrm{B}}^{\mathrm{DTI}}$ that avoids both blame caused by wrong choice of static types and the problem caused by substituting $\star$. A main idea is to allow a $\lambda_{\mathrm{B}}^{\mathrm{DTI}}$ term to contain type variables (that represent undecided ones during static-namely, usual compile-time-type inference) and defer instantiation of these type variables to run time. Specifically, we introduce dynamic type inference (DTI) into the semantics of $\lambda_{\mathrm{B}}^{\mathrm{DTI}}$ so that type variables are instantiated along reduction. For example, the term

$$
\left(\lambda x: \star .\left(x: \star \Rightarrow^{\ell_{1}} \star \rightarrow \star\right)\left(2: \text { int } \Rightarrow^{\ell_{2}} \star\right)\right)\left((\lambda y: Y . y): Y \rightarrow Y \Rightarrow^{\ell_{3}} \star\right)
$$

(obtained from $(\lambda x: \star \cdot x 2)(\lambda y \cdot y))$ reduces to

$$
\left((\lambda y: Y \cdot y)\left(2: \text { int } \Rightarrow^{\ell_{2}} \star \Rightarrow^{\bar{\ell}_{3}} Y\right)\right): Y \Rightarrow^{\ell_{3}} \star
$$

and then, instead of raising blame, it reduces to

$$
((\lambda y \text { :int. } y) 2): \text { int } \Rightarrow^{\ell_{3}} \star
$$

by instantiating $Y$ with int. (Shaded parts denote where instantiation took place.) In general, when a tagged value $w: \iota \Rightarrow^{\ell} \star$ (where $\iota$ is a base type) meets a cast to a type variable $Y$, the cast succeeds and $Y$ is instantiated to $\iota$. Similarly, if a tagged function value is cast to $Y$, two fresh type variables $Y_{1}$ and $Y_{2}$ are generated and $Y$ is instantiated to $Y_{1} \rightarrow Y_{2}$, expecting further instantiation later.

Unlike the semantics based on substitution of $\star$, the DTI-based semantics raises blame if a type variable appears in conflicting contexts. For example, the term

$$
\begin{aligned}
& \left(\lambda x: \star \rightarrow \star \rightarrow \star \cdot x\left(2: \text { int } \Rightarrow^{\ell_{1}} \star\right)\left(\text { true }: \text { bool } \Rightarrow^{\ell_{2}} \star\right)\right) \\
& \quad\left(\left(\lambda y_{1}: Y . \lambda y_{2}: Y \text {. if } b \text { then } y_{1} \text { else } y_{2}\right): Y \rightarrow Y \rightarrow Y \Rightarrow^{\ell_{3}} \star \rightarrow \star \rightarrow \star\right)
\end{aligned}
$$

(obtained from $\left(\lambda x: \star \rightarrow \star \rightarrow \star . x 2\right.$ true) $\left(\lambda y_{1}: Y . \lambda y_{2}: Y\right.$. if $b$ then $y_{1}$ else $\left.\left.y_{2}\right)\right)$ reduces in a few steps to

$$
\left(\left(\left(\lambda y_{1}: Y . \lambda y_{2}: Y \text {. if } b \text { then } y_{1} \text { else } y_{2}\right)\left(2: \text { int } \Rightarrow^{\ell_{1}} \star \Rightarrow^{\bar{\ell}_{3}} Y\right)\right): Y \rightarrow Y \Rightarrow^{\ell_{3}} \star \rightarrow \star\right) \cdots
$$

which corresponds to $x 2$ in the source term. The cast on 2 succeeds and this term reduces to $\left(\left(\lambda y_{2}\right.\right.$ : int. if $b$ then 2 else $\left.y_{2}\right):$ int $\rightarrow$ int $\left.\Rightarrow^{\ell_{3}} \star \rightarrow \star\right)$ (true : bool $\left.\Rightarrow^{\ell_{2}} \star\right)$ by instantiating $Y$ with int (where the shaded parts are the results of instantiation). Then, in the next step, reduction reaches the application of $x 2$ to true (in the source term):

$$
\left.\left(\left(\left(\lambda y_{2} \text { : int. if } b \text { then } 2 \text { else } y_{2}\right) \text { (true : bool } \Rightarrow^{\ell_{2}} \star \Rightarrow^{\bar{\ell}_{3}} \text { int }\right)\right): \text { int } \Rightarrow^{\ell_{3}} \star\right) \text {. }
$$

However, the shaded cast on true fails. In short, $Y$ is required to be both int and bool at the same time, which is impossible. As this example shows, DTI is not as permissive as the semantics based on substitution of $\star$ and detects a type error early.

DTI is sound and complete. Intuitively, soundness means that, if a program evaluates to a value under the DTI semantics, then the program obtained by applying-in advance-the type instantiation that DTI found results in the same value and if a program results in blame under the DTI semantics, 
then all type substitutions make the program result also in blame. Completeness means that, if some type substitution makes the program evaluate to a value, then execution with DTI also results in a related value. Soundness also means that the semantics is not too permissive: it is not the case that a program evaluates to a value under the DTI semantics but no type substitution makes the program evaluate to a value. The semantics based on substituting $\star$ is complete but not sound; the semantics based on "undecided type variables as base types" as in Garcia and Cimini [2015] is neither sound nor complete (because it just raises blame too often).

We equip $\lambda_{\mathrm{B}}^{\mathrm{DTI}}$ with ML-style let-polymorphism [Milner 1978]. Actually, Garcia and Cimini have already proposed two ways to implement the ITGL with let-polymorphism: by translating it to the Polymorphic Blame Calculus [Ahmed et al. 2011, 2017] or by expanding let before translating it to the (monomorphic) blame calculus. However, they have left a detailed comparison of the two to future work. Our semantics is very close to the latter, although we do not statically expand definitions by let. Perhaps surprisingly, the semantics is not quite parametric; we argue that translation to the Polymorphic Blame Calculus, which dynamically enforces parametricity [Ahmed et al. 2017; Reynolds 1983], has an undesirable consequence and our semantics (which is close to the one based on expanding let) is better suited for languages in which type abstraction and application are implicit.

Other than soundness and completeness of DTI, we also study the gradual guarantee property [Siek et al. 2015a] for the ITGL. The gradual guarantee formalizes an informal expectation for gradual typing systems that adding more static types to a program only exposes type errorswhether they are static or dynamic-but should not change the behavior of the program otherwise. To deal with the ITGL, where bound variables come with optional type annotations, we extend the notion of "more static types" (formalized as precision relations $\sqsubseteq$ over types and terms) so that an omitted type annotation is more precise than the annotation with $\star$ but less precise than an annotation with a static type. So, for example,

$$
(\lambda x: \text { int } \rightarrow \text { int. } x 2)(\lambda y: \text { int. } y) \sqsubseteq(\lambda x .2)(\lambda y: \text { int. } y) \sqsubseteq(\lambda x: \star .2)(\lambda y: \star . y) .
$$

Intuitively, omitted type annotations are considered (fresh) type variables, which are less specific than concrete types but they are more precise than $\star$ because they range only over static types. We prove the gradual guarantee for the ITGL. To our knowledge, the gradual guarantee is proved for a language with let-polymorphism for the first time.

Finally, we have implemented an interpreter of the ITGL, including an implementation of Garcia and Cimini's type inference algorithm, a translator to and an evaluator of $\lambda_{\mathrm{B}}^{\mathrm{DTI}}$, in OCaml. It supports integer, Boolean, and unit types as base types, standard arithmetic, comparison, and Boolean operators, conditional expressions, and recursive definitions. The source code is available at https://github.com/ymyzk/lambda-dti/.

Contributions. Our contributions are summarized as follows:

- We propose DTI as a basis for new semantics of (an intermediate language for) the ITGL, an implicitly typed language with a gradual type system and Hindley-Milner polymorphism;

- We define a blame calculus $\lambda_{\mathrm{B}}^{\mathrm{DTI}}$ with its syntax, type system, and operational semantics with DTI;

- We prove properties of $\lambda_{\mathrm{B}}^{\mathrm{DTI}}$, including type safety, soundness and completeness of DTI, and the gradual guarantee;

- We also prove the gradual guarantee for the ITGL; and

- We have implemented an interpreter of the ITGL.

The organization of the paper. We define $\lambda_{\mathrm{B}}^{\mathrm{DTI}}$ in Section 2 and state its basic properties, including type safety and conservative extension, in Section 3 . Then, we show soundness and completeness 


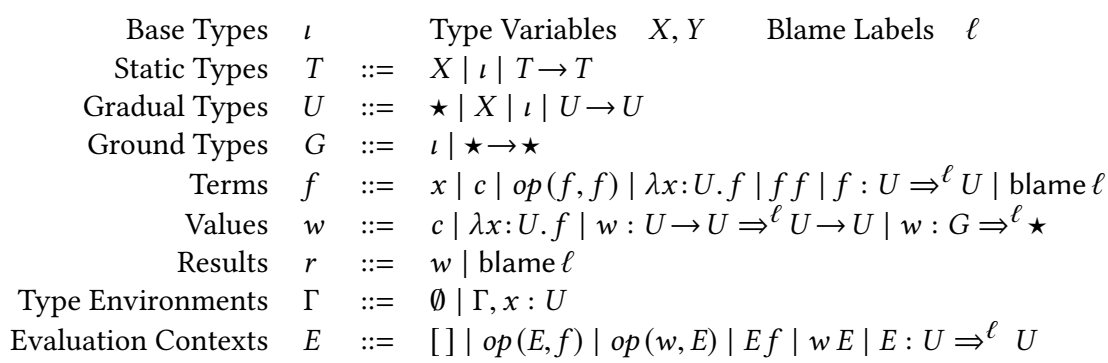

Fig. 1. Syntax of $\lambda_{\mathrm{B}}^{\mathrm{DTI}}$.

of DTI in Section 4 and the gradual guarantee in Section 5. Finally, we discuss related work in Section 6 and conclude in Section 7. All the stated properties have been proved but proofs are mostly omitted; see the full version at http://arxiv.org/abs/1810.12619.

\section{$2 \lambda_{\mathbf{B}}^{\text {DTI }}$ : A BLAME CALCULUS WITH DYNAMIC TYPE INFERENCE}

In this section, we develop a new blame calculus $\lambda_{\mathrm{B}}^{\mathrm{DTI}}$ with dynamic type inference (DTI). We start with a simply typed fragment and add let-polymorphism in Section 2.3. The core of the $\lambda_{\mathrm{B}}^{\mathrm{DTI}}$ calculus is based on the calculus by Siek et al. [2015a], which is a simplified version of the blame calculus by Wadler and Findler [2009] without refinement types. We augment its type system with type variables in a fairly straightforward manner and operational semantics as described in the last section.

Our blame calculus $\lambda_{\mathrm{B}}^{\mathrm{DTI}}$ is designed to be used as an intermediate language to give semantics of the ITGL by Garcia and Cimini [2015]. We will discuss the ITGL and how ITGL programs are translated to $\lambda_{\mathrm{B}}^{\mathrm{DTI}}$ programs in Section 5 in more detail, but as far as the simply typed fragment is concerned it is very similar to previous work [Garcia and Cimini 2015; Siek et al. 2015a].

\subsection{Static Semantics}

We show the syntax of $\lambda_{\mathrm{B}}^{\mathrm{DTI}}$ in Figure 1 . The syntax of the calculus extends that of the simply typed lambda calculus with the dynamic type, casts, and type variables.

Gradual types, denoted by $U$, consist of base types $\iota$ (such as int and bool), type variables $X$, the dynamic type $\star$, and function types $U \rightarrow U$. Static types, denoted by $T$, are the subset of gradual types without the dynamic type. Ground types, which are used as tags to inject values into the dynamic type, contain base types $\iota$ and the function type $\star \rightarrow \star$. We emphasize that type variables are not in ground types, because no value inhabits them (as we show in the canonical forms lemma (Lemma 3)) and we do not need to use a type variable as a tag to inject values.

Terms, denoted by $f$, consist of variables $x$, constants $c$, primitive operations op $(f, f)$, lambda abstractions $\lambda x: U . f$ (which bind $x$ in $f$ ), applications $f f$, casts $f: U \Rightarrow^{\ell} U$, and blame blame $\ell$. Since this is an intermediate language, variables in abstractions are explicitly typed. Casts $f: U_{1} \Rightarrow \ell$ $U_{2}$ from $U_{1}$ to $U_{2}$ are inserted when translating a term in the ITGL, and used for checking whether $f$ of type $U_{1}$ can behave as type $U_{2}$ at run time. Casts are annotated also with blame labels, denoted by $\ell$, to indicate which cast has failed; blame blame $\ell$ is used to denote a run-time failure of a cast with $\ell$. They have polarity to indicate which side of a cast to be blamed [Findler and Felleisen 2002]. For each blame label, there is a negated blame label $\bar{\ell}$, which denotes the opposite side to $\ell$, and $\overline{\bar{\ell}}=\ell$. As we did in the introduction, we often abbreviate a sequence of casts $\left(f: U_{1} \Rightarrow^{\ell_{1}} U_{2}\right): U_{2} \Rightarrow^{\ell_{2}} U_{3}$ to $f: U_{1} \Rightarrow^{\ell_{1}} U_{2} \Rightarrow^{\ell_{2}} U_{3}$. 
Type consistency: $U \sim U$

$$
\begin{aligned}
& \overline{\iota \sim \iota} \text { C_BAse } \quad \overline{X \sim X} \text { C_TyVar } \quad \overline{\star \sim U} \text { C_DynL } \overline{U \sim \star} \text { C_DynR } \\
& \frac{U_{11} \sim U_{21} \quad U_{12} \sim U_{22}}{U_{11} \rightarrow U_{12} \sim U_{21} \rightarrow U_{22}} \text { C_ARROW }
\end{aligned}
$$

Typing rules: $\Gamma \vdash f: U$

$$
\begin{aligned}
& \frac{x: U \in \Gamma}{\Gamma \vdash x: U} \text { T_VAR } \quad \frac{t y(o p)=\iota_{1} \rightarrow \iota_{2} \rightarrow \iota \quad \Gamma \vdash f_{1}: \iota_{1} \quad \Gamma \vdash f_{2}: \iota_{2}}{\Gamma \vdash c: t y(c)} \text { T_Const } \quad \text { T_Op } \\
& \frac{\Gamma, x: U_{1} \vdash f: U_{2}}{\Gamma \vdash \lambda x: U_{1} \cdot f: U_{1} \rightarrow U_{2}} \text { T_ABS } \quad \frac{\Gamma \vdash f_{1}: U_{1} \rightarrow U_{2} \quad \Gamma \vdash f_{2}: U_{1}}{\Gamma \vdash f_{1} f_{2}: U_{2}} \text { T_ApP } \\
& \frac{\Gamma \vdash f: U \quad U \sim U^{\prime}}{\Gamma \vdash\left(f: U \Rightarrow^{\ell} U^{\prime}\right): U^{\prime}} \text { T_CAST } \quad \frac{}{\Gamma \vdash \text { blame } \ell: U} \text { T_BLAME }
\end{aligned}
$$

Fig. 2. Static semantics of $\lambda_{\mathrm{B}}^{\mathrm{DTI}}$.

Values, denoted by $w$, consist of constants, lambda abstractions, wrapped functions, and injections. A wrapped function is a function value enclosed in the cast between function types. Results, denoted by $r$, are values and blame. Evaluation contexts, denoted by $E$, are standard and they mean that a term is evaluated from left to right, under call-by-value.

We show the static semantics of $\lambda_{\mathrm{B}}^{\mathrm{DTI}}$ in Figure 2. It consists of type consistency and typing.

Type consistency rules define the type consistency relation $U \sim U^{\prime}$, a key notion in gradual typing, over gradual types. Intuitively, $U \sim U^{\prime}$ means that it is possible for a cast from $U$ to $U^{\prime}$ to succeed. The rules (C_BASE) and (C_TYVAR) mean that a base type and a type variable, respectively, is consistent with itself. The rules (C_DynL) and (C_DynR) mean that all gradual types are consistent with the dynamic type. The rule (C_ARrow) means that two function types are consistent if their domain types are consistent and so are their range types. The type consistency relation is reflexive and symmetric but not transitive.

Typing rules of the $\lambda_{\mathrm{B}}^{\mathrm{DTI}}$ extend those of the simply typed lambda calculus. The rules (T_VAR), (T_Const), (T_Op), (T_ABs), and (T_App) are standard. $t y(c)$ used in (T_Const) assigns a base type to each constant $c$, and $t y(o p)$ used in (T_Op) assigns a first-order static type without type variables to each operator op. The rule (T_CAST) allows a term to be cast to a consistent type.

A type substitution, denoted by $S$, is a finite mapping from type variables to static types. The empty mapping is denoted by []; and the composition of two type substitutions $S_{1}$ and $S_{2}$ is by $S_{1} \circ S_{2}$. Application $S(f)$ and $S(U)$ of type substitution $S$ to term $f$ and type $U$ are defined in the usual way respectively. We write $[\vec{X}:=\vec{T}]$ for a type substitution that maps type variables $\vec{X}$ to types $\vec{T}$ respectively and $f[\vec{X}:=\vec{T}]$ and $U[\vec{X}:=\vec{T}]$ for $[\vec{X}:=\vec{T}](f)$ and $[\vec{X}:=\vec{T}](U)$. Note that the codomain of a type substitution is static types, following Garcia and Cimini [2015], in which a type variable represents a placeholder for a static type.

As expected, type substitution preserves consistency and typing:

Lemma 1 (Type Substitution Preserves Consistency and Typing).

(1) If $U \sim U^{\prime}$, then $S(U) \sim S\left(U^{\prime}\right)$ for any $S$.

(2) If $\Gamma \vdash f: U$, then $S(\Gamma) \vdash S(f): S(U)$ for any $S$. 

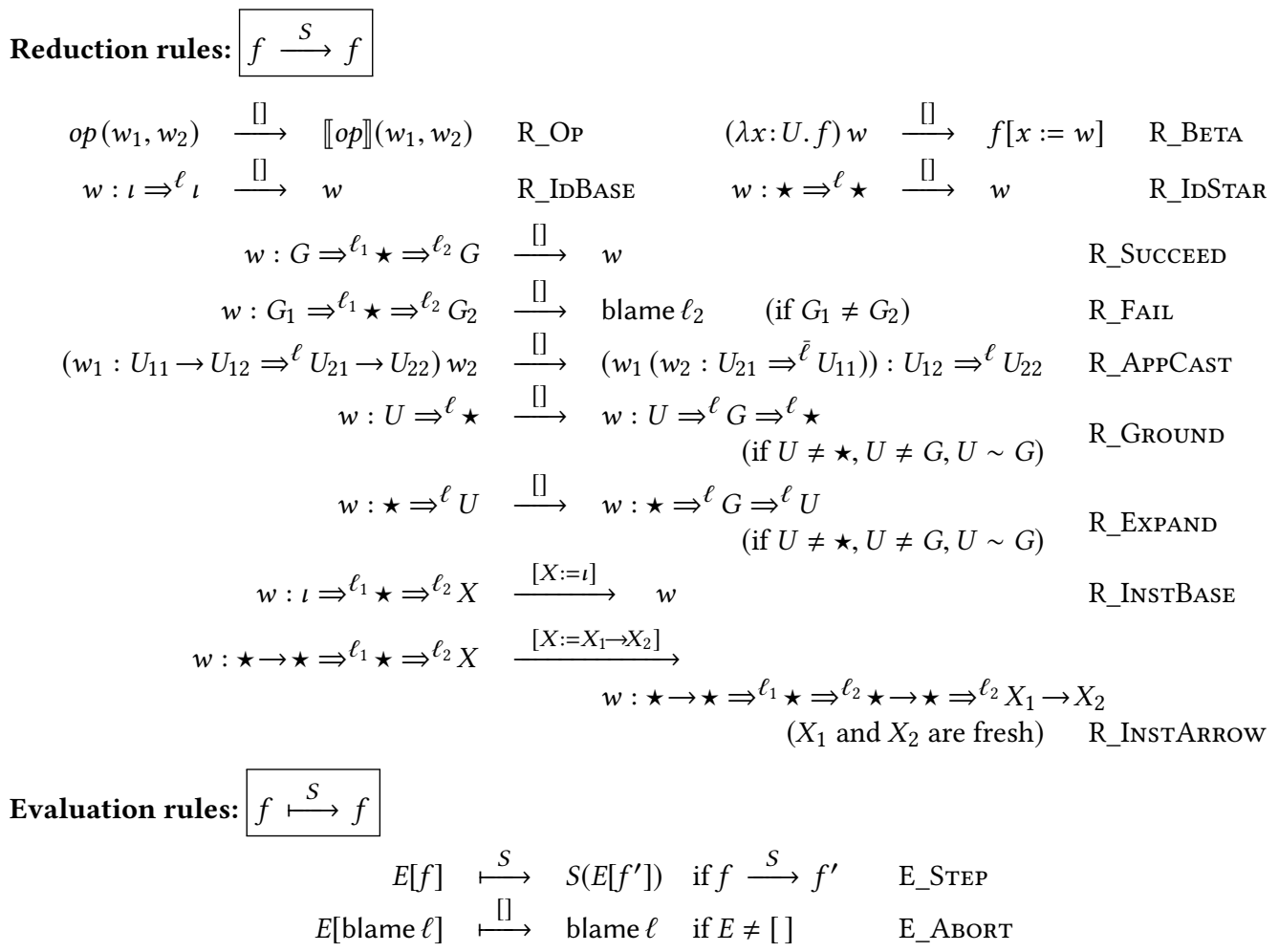

Fig. 3. Dynamic semantics of $\lambda_{\mathrm{B}}^{\mathrm{DTI}}$.

\subsection{Dynamic Semantics}

We show the dynamic semantics of $\lambda_{\mathrm{B}}^{\mathrm{DTI}}$ in Figure 3. It is given in a small-step style by using two relations over terms. One is the reduction relation $f \stackrel{S}{\longrightarrow} f^{\prime}$, which represents a basic computation step, including dynamic checking by casts and DTI. The other is the evaluation relation $f \stackrel{S}{\longmapsto} f^{\prime}$, which represents top-level execution. Both relations are annotated with a type substitution $S$, which is generated by DTI.

2.2.1 Basic Reduction Rules. We first explain rules from the basic blame calculus, where type

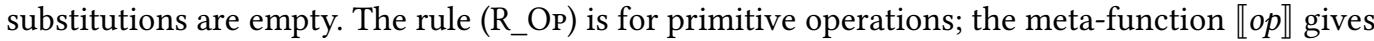
a meaning to the primitive operation $o p$ and we assume that $\llbracket o p \rrbracket\left(w_{1}, w_{2}\right)$ returns a value of the right type, i.e., the return type of $t y(o p)$. The rule (R_BETA) performs the standard $\beta$-reduction. We write $f[x:=w]$ for the term obtained by substituting $w$ for $x$ in $f$; term substitution is defined in a capture-avoiding manner as usual. The rules (R_IDBASE) and (R_IDSTAR) discard identity casts on a base type and the dynamic type, respectively. The rules (R_SUCCEED) and (R_FAIL) check two casts where an injection (a cast from a ground type to the dynamic type) meets a projection (a cast from the dynamic type to a ground type). If both ground types are equal, then the projection succeeds and these casts are discarded. Otherwise, these casts fail and reduce to blame to abort execution of the program with blame label $\ell_{2}$ (the one on the projection). The rule (R_ApPCAST) reduces an application of a wrapped function by breaking the cast into two. One is a cast on the argument, and the other is a cast on the return value. We negate the blame label for the cast on the argument 
because the direction of the cast is swapped from that of the function cast [Findler and Felleisen 2002]. The rules (R_Ground) and (R_ExPAND) decompose a cast between a non-ground type and the dynamic type into two casts. These rules cannot be used if $U$ is a type variable because type variables are never consistent with any ground type. The ground type in the middle of the resulting two casts is uniquely determined:

LEMma 2 (Ground Types).

(1) If $U$ is neither a type variable nor the dynamic type, then there exists a unique $G$ such that $U \sim G$.

(2) $G \sim G^{\prime}$ if and only if $G=G^{\prime}$.

2.2.2 Reduction Rules for Dynamic Type Inference. As discussed in the introduction, our idea is to infer the "value" of a type variable when it is projected from the dynamic type, as in $w: \star \Rightarrow^{\ell} X$. The value typed at the dynamic type is always tagged with a ground type and is of the form $w^{\prime}: G \Rightarrow^{\ell^{\prime}} \star$. If the ground type $G$ is a base type, then the type variable will be instantiated with it. We revisit the example used in Section 1 and show evaluation steps below.

$$
\begin{aligned}
& \left(\lambda x: \star .\left(x: \star \Rightarrow^{\ell_{1}} \star \rightarrow \star\right)\left(2: \text { int } \Rightarrow^{\ell_{2}} \star\right)\right)\left((\lambda y: Y \cdot y): Y \rightarrow Y \Rightarrow^{\ell_{3}} \star\right) \\
& \stackrel{[]}{\longmapsto}^{*} \quad\left((\lambda y: Y \cdot y): Y \rightarrow Y \Rightarrow^{\ell_{3}} \star \rightarrow \star\right)\left(2: \text { int } \Rightarrow^{\ell_{2}} \star\right) \\
& \stackrel{[]}{\longmapsto} \quad\left((\lambda y: Y \cdot y)\left(2: \text { int } \Rightarrow^{\ell_{2}} \star \Rightarrow^{\bar{\ell}_{3}} Y\right)\right): Y \Rightarrow^{\ell_{3}} \star \\
& \stackrel{[Y:=\text { int }]}{\longrightarrow}((\lambda y \text { :int. } y) 2): \text { int } \Rightarrow^{\ell_{3}} \star \\
& \stackrel{[]}{\longmapsto} \quad 2: \text { int } \Rightarrow^{\ell_{3}} \star
\end{aligned}
$$

The subterm $2:$ int $\Rightarrow^{\ell_{2}} \star \Rightarrow^{\bar{\ell}_{3}} Y$ on the third line reduces to 2-this is where DTI is performed; this reduction step is annotated with a type substitution $[Y:=$ int $]$, which roughly means " $Y$ must be int for the execution of the program to proceed further without blame." As we will explain soon, the type substitution is applied to the whole term, since the type variable $Y$ may appear elsewhere in the term. As a result, the occurrences of $Y$ in the type annotation and the last cast are also instantiated (as the shade on int indicates) and so $\left((\lambda y: Y . y)\left(2:\right.\right.$ int $\left.\left.\Rightarrow^{\ell_{2}} \star \Rightarrow^{\bar{\ell}_{3}} Y\right)\right): Y \Rightarrow^{\ell_{3}} \star$ evaluates to $((\lambda y$ :int. $y) 2)$ : int $\Rightarrow^{\ell_{3}} \star$ in one step.

Now, we explain formal reduction rules for DTI. The rule (R_InstBASE) instantiates a type variable $X$ with the base type $\iota$ and generates a type substitution $[X:=\imath]$. The rule (R_InstArrow) instantiates a type variable $X$ with a function type $X_{1} \rightarrow X_{2}$ for fresh type variables $X_{1}$ and $X_{2}{ }^{2}$ At this point, we know that $w$ is a (possibly wrapped) function, but the domain and range types are still unknown. We defer the decision about these types by generating fresh type variables, which will be instantiated in the future evaluation.

Finally, we explain the evaluation rules. The rule (E_STEP) reduces the subterm in an evaluation context, then apply the generated type substitution. The substitution is applied to the whole term $E\left[f^{\prime}\right]$ so that the other occurrences of the same variables are replaced at once. The rule (E_ABORT) aborts execution of a program if it raises blame.

We write $f_{0} \stackrel{S}{\longmapsto} * f_{n}$ if $f_{0} \stackrel{S_{1}}{\longmapsto} f_{1}, f_{1} \stackrel{S_{2}}{\longmapsto} f_{2}, \ldots$, and $f_{n-1} \stackrel{S_{n}}{\longmapsto} f_{n}$ and $S=S_{n} \circ S_{n-1} \circ \cdots \circ S_{1}$ (where $n \geq 0$ ) and similarly for $f_{0} \stackrel{S}{\longmapsto} f_{n}$ (where $n \geq 1$ ).

One may wonder that the rule (R_InsTARrow) is redundant because a term $w: \star \rightarrow \star \Rightarrow^{\ell_{1}}$ $\star \Rightarrow^{\ell_{2}} \star \rightarrow \star \Rightarrow^{\ell_{2}} X_{1} \rightarrow X_{2}$ always reduces to $w: \star \rightarrow \star \Rightarrow^{\ell_{2}} X_{1} \rightarrow X_{2}$ in the next step. Actually, we could define this reduction rule in the following two different manners:

${ }^{2}$ We use the term "fresh" here to mean that $X_{1}$ and $X_{2}$ occur nowhere in the whole program before reduction. 
- $w: \star \rightarrow \star \Rightarrow^{\ell_{1}} \star \Rightarrow^{\ell_{2}} X \stackrel{\left[X:=X_{1} \rightarrow X_{2}\right]}{\longrightarrow} w: \star \rightarrow \star \Rightarrow^{\ell_{2}} X_{1} \rightarrow X_{2}$; or
- $w: \star \rightarrow \star \Rightarrow^{\ell_{1}} \star \Rightarrow^{\ell_{2}} X \stackrel{\left[X:=X_{1} \rightarrow X_{2}\right]}{\longrightarrow} w: \star \rightarrow \star \Rightarrow^{\ell_{1}} \star \Rightarrow^{\ell_{2}} X_{1} \rightarrow X_{2}$.

Using these rules does not change the semantics of the language, but we choose (R_InstARROw) for ease of proofs.

We show how the rule (R_InstARROw) works using the following (somewhat contrived ${ }^{3}$ ) example:

$$
\begin{aligned}
& \left((\lambda y \text { : int. } y+1): \text { int } \rightarrow \text { int } \Rightarrow^{\ell_{1}} \star \Rightarrow^{\ell_{2}} X \Rightarrow^{\ell_{3}} \star \Rightarrow^{\ell_{4}} \star \rightarrow \star\right)\left(3 \text { : int } \Rightarrow^{\ell_{5}} \star\right) \\
& \stackrel{[]}{\longmapsto} \quad\left(w: \star \rightarrow \star \Rightarrow^{\ell_{1}} \star \Rightarrow^{\ell_{2}} X \Rightarrow^{\ell_{3}} \star \Rightarrow^{\ell_{4}} \star \rightarrow \star\right)\left(3 \text { : int } \Rightarrow^{\ell_{5}} \star\right) \\
& \text { where } w=(\lambda y \text { :int. } y+1) \text { : int } \rightarrow \text { int } \Rightarrow^{\ell_{1}} \star \rightarrow \star \\
& \stackrel{S}{\longmapsto} \quad\left(w: \star \rightarrow \star \Rightarrow^{\ell_{1}} \star \Rightarrow^{\ell_{2}} \star \rightarrow \star \Rightarrow^{\ell_{2}} X_{1} \rightarrow X_{2} \Rightarrow^{\ell_{3}} \star \Rightarrow^{\ell_{4}} \star \rightarrow \star\right)\left(3: \text { int } \Rightarrow^{\ell_{5}} \star\right) \\
& \text { where } S=\left[X:=X_{1} \rightarrow X_{2}\right] \\
& \stackrel{S}{\prime}^{*} \quad 4: \text { int } \Rightarrow^{\ell_{4}} \star \quad \text { where } S^{\prime}=\left[X_{1}:=\text { int, } X_{2}:=\text { int }\right] .
\end{aligned}
$$

The type variable $X$ is instantiated with $X_{1} \rightarrow X_{2}$ when the value $w$ tagged with $\star \rightarrow \star$ is projected to $X$. Then, $X_{1}$ and $X_{2}$ are instantiated with int by (R_InstBASE), as we have already explained, by the time the term evaluates to the final value $4:$ int $\Rightarrow^{\ell_{4}} \star$.

Perhaps surprisingly, our reduction rules to instantiate type variables are not symmetric: There are no rules to reduce terms such as $w: X \Rightarrow^{\ell_{1}} \star \Rightarrow^{\ell_{2}} \iota, w: X \Rightarrow^{\ell_{1}} \star \Rightarrow^{\ell_{2}} \star \rightarrow \star, w: X \Rightarrow^{\ell_{1}} \star \Rightarrow^{\ell_{2}} X^{\prime}$, and $w: X \Rightarrow^{\ell_{1}} X$, even though a cast expression such as $f: X \Rightarrow^{\ell} \star$ does appear during reduction. This is because a value is not typed at a type variable as we will show in the canonical forms lemma (Lemma 3) and we do not need the rules to reduce these terms. The $X$ in $f: X \Rightarrow^{\ell} \star$ will be instantiated during evaluation of $f$.

Before closing this subsection, we revisit an example from the introduction. It raises blame because one type variable is used in contexts that expect different types:

$$
\left(\lambda x: \star \rightarrow \star \rightarrow \star \cdot x\left(2: \text { int } \Rightarrow^{\ell_{1}} \star\right)\left(\text { true }: \text { bool } \Rightarrow^{\ell_{2}} \star\right)\right) w
$$

where $w=\left(\lambda y_{1}: Y . \lambda y_{2}: Y\right.$. if $b$ then $y_{1}$ else $\left.y_{2}\right): Y \rightarrow Y \rightarrow Y \Rightarrow^{\ell_{3}} \star \rightarrow \star \rightarrow \star$

$\stackrel{[]}{\longmapsto} \quad w\left(2:\right.$ int $\left.\Rightarrow^{\ell_{1}} \star\right)\left(\right.$ true : bool $\left.\Rightarrow^{\ell_{2}} \star\right)$

$\stackrel{[]}{\longmapsto} \quad\left(\left(\left(\lambda y_{1}: Y \cdot \lambda y_{2}: Y\right.\right.\right.$. if $b$ then $y_{1}$ else $\left.y_{2}\right)\left(2:\right.$ int $\left.\left.\left.\Rightarrow^{\ell_{1}} \star \Rightarrow^{\bar{\ell}_{3}} Y\right)\right): Y \rightarrow Y \Rightarrow^{\ell_{3}} \star \rightarrow \star\right)$

(true : bool $\Rightarrow^{\ell_{2}} \star$ )

$\stackrel{[Y:=\text { int }]}{\longmapsto}\left(\left(\left(\lambda y_{1}\right.\right.\right.$ :int. $\lambda y_{2}$ :int. if $b$ then $y_{1}$ else $\left.\left.y_{2}\right) 2\right):$ int $\rightarrow$ int $\left.\Rightarrow^{\ell_{3}} \star \rightarrow \star\right)\left(\right.$ true $:$ bool $\left.\Rightarrow^{\ell_{2}} \star\right)$

$\longmapsto^{*} \quad\left(\left(\lambda y_{2}\right.\right.$ : int. if $b$ then 2 else $\left.y_{2}\right)$ (true : bool $\Rightarrow^{\ell_{2}} \star \Rightarrow^{\bar{\ell}_{3}}$ int $\left.)\right):$ int $\Rightarrow^{\ell_{3}} \star$

$\longmapsto \quad$ blame $\bar{\ell}_{3}$

As for the first example, at the third step, the subterm $\left(2:\right.$ int $\left.\Rightarrow^{\ell_{1}} \star \Rightarrow^{\bar{\ell}_{3}} Y\right)$ reduces to 2 by (R_InstBASE) and a substitution $[Y:=$ int] is yielded. Then, by application of (E_STEP), $Y$ in the evaluation context also gets replaced with int. (Again, the shaded types indicate which parts are affected.) This term eventually evaluates to blame because the cast (true : bool $\Rightarrow^{\ell_{2}} \star \Rightarrow^{\bar{\ell}_{3}}$ int) fails. After all, the function $\lambda y_{1} . \lambda y_{2}$. if $b$ then $y_{1}$ else $y_{2}$ cannot be used as both int $\rightarrow$ int $\rightarrow$ int and bool $\rightarrow$ bool $\rightarrow$ bool at the same time. It is important to ensure that $Y$ is instantiated at most once.

\footnotetext{
${ }^{3}$ In fact, this term is not in the image of the cast-inserting translation. An example in the image would be much more complicated.
} 


\subsection{Let-Polymorphism}

In this section, we extend $\lambda_{\mathrm{B}}^{\mathrm{DTI}}$ to let-polymorphism [Milner 1978] by introducing type schemes and explicit type abstraction $\Lambda \vec{X} . w$ and application $x[\vec{T}]$, as Core-XML [Harper and Mitchell 1993]. (Here, we abbreviate sequences by using vector notations.) Explicit type abstraction/application is needed because we need type information at run time.

Our formulation is fairly standard but we will find a few twists that are motivated by our working hypothesis that let $x=w$ in $f$ (in the surface language) should behave, both statically and dynamically, the same as $f$ where $w$ is substituted for $x$-especially in languages where type abstractions and applications are implicit. Consider the following expression in the surface language (extended with pairs):

$$
\text { let } g=\lambda x .((\lambda y \cdot y):: \star \rightarrow \star) x \text { in }(g 2, g \text { true })
$$

where $(\lambda y \cdot y):: \star \rightarrow \star$ stands for ascription, which is translated to a cast. By making casts, type abstraction, and type application explicit, one would obtain something like

$$
\text { let } g=\Lambda X Y . \lambda x: X .\left((\lambda y: Y . y): Y \rightarrow Y \Rightarrow^{\ell} \star \rightarrow \star\right) x \text { in }\left(g\left[T_{1}, T_{2}\right] 2, g\left[T_{3}, T_{4}\right] \text { true }\right)
$$

in $\lambda_{\mathrm{B}}^{\mathrm{DTI}}$. Note that, due to the use of $\star \rightarrow \star$, different type variables are assigned to $x$ and $y$ and so $g$ is bound to a two-argument type abstraction. Now, what should type arguments be at the two uses of $g$ ? It should be obvious that $T_{1}$ and $T_{3}$ have to be int and bool, respectively, from arguments to $g$. At first, it may seem that $T_{2}$ and $T_{4}$ should be int and bool, respectively, in order to avoid blame. However, it is hard for a type system to see it-in fact, $g$ is given type scheme $\forall X Y . X \rightarrow \star$ and, since $Y$ is bound but not referenced, there is no clue. We assign a special symbol $v$ to $T_{2}$ and $T_{4}$; each occurrence of $v$ is replaced with a fresh type variable when a (polymorphic) value is substituted for $g$ during reduction. These fresh type variables are expected to be instantiated by DTI-to int and bool in this example-as reduction proceeds.

$$
\begin{aligned}
& \text { let } g=\Lambda X Y . \lambda x: X .\left((\lambda y: Y . y): Y \rightarrow Y \Rightarrow^{\ell} \star \rightarrow \star\right) x \text { in }(g[\text { int, } v] 2, g[\text { bool, } v] \text { true }) \\
& \left(\left(\lambda x: \text { int. }\left(\left(\lambda y: Y_{1} \cdot y\right): Y_{1} \rightarrow Y_{1} \Rightarrow^{\ell} \star \rightarrow \star\right) x\right) 2\right. \text {, } \\
& \left.\left(\lambda x: \text { bool. }\left(\left(\lambda y: Y_{2} \cdot y\right): Y_{2} \rightarrow Y_{2} \Rightarrow^{\ell} \star \rightarrow \star\right) x\right) \text { true }\right)
\end{aligned}
$$

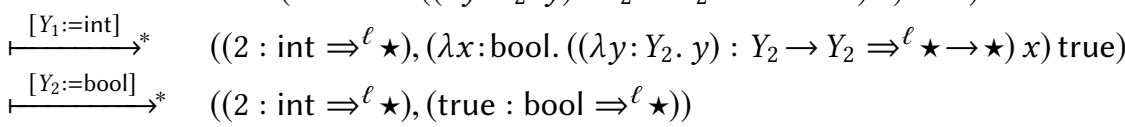

We do not assign fresh type variables when type abstractions/applications are made explicit during cast insertion. It is because an expression, such as $g\left[T_{1}, T_{3}\right]$, including type applications may be duplicated during reduction and sharing a type variable among duplicated expressions might cause unwanted blame.

Figure 4 shows the definition of the extension. A type scheme, denoted by $\sigma$, is a gradual type abstracted over a (possibly empty) finite sequence of type variables, denoted by $\vec{X}_{i}$. A type environment is changed so that it maps variables to type schemes, instead of gradual types. Terms are extended with let-expressions of the form let $x=\Lambda \vec{X}_{i}$. $w$ in $f$, which bind $\vec{X}_{i}$ in value $w$, and variables $x\left[\overrightarrow{\mathbb{T}_{i}}\right]$, which represent type application if $x$ is let-bound, are now annotated with a sequence of type arguments. ${ }^{5}$ A type argument is either a static type $T$ or the special symbol $v$. Type substitution is defined in a capture-avoiding manner.

We adopt value restriction [Wright 1995], i.e., the body of a type abstraction has to be a syntactic value, for avoiding a subtle issue in the dynamic semantics. If we did not adopt value restriction, we would have to deal with cast applications where a target type is a bound type variable. For example,

\footnotetext{
${ }^{4}$ A monomorphic definition let $x=f_{1}$ in $f_{2}$ (where $f_{1}$ is not necessarily a value) can be expressed as $\left(\lambda x\right.$. $\left.f_{2}\right) f_{1}$ as usual.

${ }^{5} \mathrm{~A}$ variable introduced by a lambda abstraction is always monomorphic, so $\overrightarrow{\mathbb{T}}_{i}$ is empty for such a variable.
} 
Syntax:

$$
\begin{array}{rlll}
\text { Type Schemes } & \sigma & := & U \mid \forall X . \sigma \\
\text { Type Environments } & \Gamma & := & \emptyset \mid \Gamma, x: \sigma \\
\text { Type Arguments } & \mathbb{T} & ::= & T \mid v \\
\text { Terms } & f & ::= & \cdots\left|x\left[\overrightarrow{\mathbb{T}}_{i}\right]\right| \text { let } x=\Lambda \vec{X}_{i} . w \text { in } f
\end{array}
$$

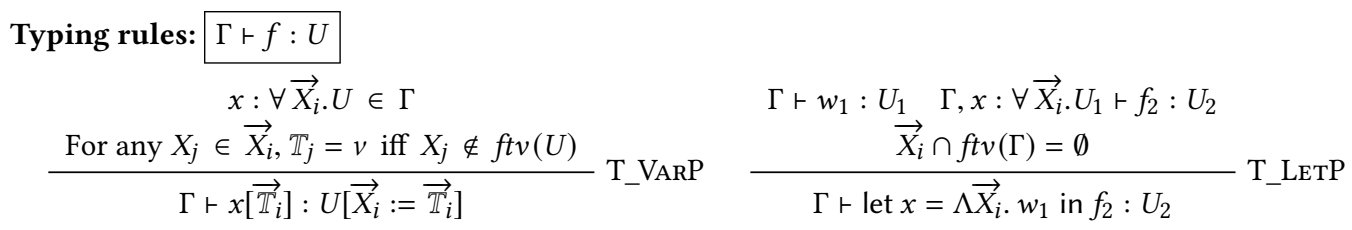

Reduction rule: $f \stackrel{S}{\longrightarrow} f$

$$
\text { let } x=\Lambda \vec{X}_{i} \text {. w in } f \stackrel{[]}{\longrightarrow} f\left[x:=\Lambda \vec{X}_{i} \cdot w\right] \quad \text { R_LETP }
$$

Substitution: $f\left[x:=\Lambda \vec{X}_{i} \cdot w\right]$

$$
\begin{aligned}
& \left(x\left[\overrightarrow{\mathbb{T}}_{i}\right]\right)\left[x:=\Lambda \vec{X}_{i} \cdot w\right]=\left[\vec{X}_{i}:=\vec{T}_{i}\right](w) \quad \text { if }\left|\vec{X}_{i}\right|=\left|\overrightarrow{\mathbb{T}}_{i}\right| \text { and } T_{i}= \begin{cases}Y_{i} & \mathbb{T}_{i}=v \text { and } Y_{i} \text { is fresh } \\
\mathbb{T}_{i} & \text { otherwise }\end{cases} \\
& \left(x^{\prime}\left[\overrightarrow{\mathbb{T}}_{i}\right]\right)\left[x:=\Lambda \vec{X}_{i} \cdot w\right]=x^{\prime}\left[\overrightarrow{\mathbb{T}}_{i}\right] \quad \text { if } x \neq x^{\prime}
\end{aligned}
$$

Fig. 4. $\lambda_{\mathrm{B}}^{\mathrm{DTI}}$ with polymorphic let.

consider the term let $x=\Lambda X$. w : int $\Rightarrow^{\ell_{1}} \star \Rightarrow^{\ell_{2}} X$ in $f$, which is actually ill formed in our language because value restriction is violated. It has a cast with bound $X$ as its target type. The question is how the cast $w$ : int $\Rightarrow^{\ell_{1}} \star \Rightarrow^{\ell_{2}} X$ is evaluated. We should not apply (R_InstBAsE) here because the type of $x$ would change from $\forall X . X$ to $\forall X$.int. It appears that there is no reasonable way to reduce this cast further-after all, the semantics of the cast depends on what type is substituted for $X$, which may be instantiated in many ways in $f$. Value restriction resolves this issue: ${ }^{6}$ a cast with its target type being a bound type variable is executed only after the type variable is instantiated.

The typing rules of $\lambda_{\mathrm{B}}^{\mathrm{DTI}}$ are also updated. We replace the rule for variables with the rule (T_VARP) and add the rule (T_LETP). The rule (T_LETP) is standard; it allows generalization by type variables that do not appear free in $\Gamma$. Note that it allows abstraction by a type variable $X$ even when $X$ does not appear in $U_{1}$. As we have already seen such abstraction can be significant in $\lambda_{\mathrm{B}}^{\mathrm{DTI}}$. The second premise of the rule (T_VARP), which represents type application, means that extra type variables that do not appear in $U$ have to be instantiated by $v$ (and other type variables by static types) The type expression $U\left[\vec{X}_{i}:=\overrightarrow{\mathbb{T}}_{i}\right]$ is notational abuse but the result will not contain $v$ because the corresponding type variables do not appear in $U$.

The rule (R_LETP) is an additional rule to reduce let-expressions. Roughly speaking, let $x=$ $\Lambda \vec{X}_{i}$. $w$ in $f$ reduces to $f$ in which $w$ is substituted for $x$ as usual but, due to explicit type abstraction/application, the definition of substitution is slightly peculiar: When a variable is replaced with a type-abstracted value, type arguments $\overrightarrow{\mathbb{T}}_{i}$ are also substituted, after fresh type variable generation, for $\vec{X}_{i}$ in the value. We formalize this idea as substitution of the form $f\left[x:=\Lambda \vec{X}_{i} . w\right]$, shown in the lower half of Figure 4; in the case for variables, the length of a sequence $\vec{X}_{i}$ or $\vec{T}_{i}$ is denoted by $|\cdot|$. This nonstandard substitution makes correspondence to usual reduction for let

\footnotetext{
${ }^{6}$ Making let call-by-name [Leroy 1993] may be another option.
} 
(that is, let $x=w$ in $f \longrightarrow f[x:=w]$ ) easier to see. Other reduction and evaluation rules, including (R_InstBAsE) and (R_InstARrow), remain unchanged.

\subsection{Discussion about the Semantics of Let-Polymorphism}

Before proceeding further, we give a brief comparison with Garcia and Cimini [2015], who have suggested that the Polymorphic Blame Calculus (PBC) [Ahmed et al. 2011, 2017] can be used to give the semantics of let in the ITGL. Using the PBC-style semantics for type abstraction/application would, however, raise more of blame-because the PBC enforces parametricity at run time-even when we just give subterms names by let.

The difference between our approach and the PBC-based one is exemplified by the following program in the ITGL:

$$
(\lambda x .1+((\lambda y: \star . y) x)) 2
$$

This program is translated into $\lambda_{\mathrm{B}}^{\mathrm{DTI}}$ and evaluated as follows:

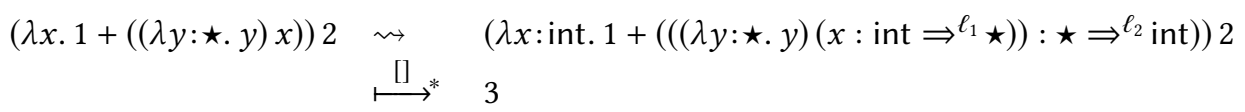

This term evaluates to a constant 3 , as expected. Next, let us rewrite this program so that it uses let to give a name to the function $\lambda x .1+((\lambda y: \star . y) x)$. Then, this program is translated into $\lambda_{\mathrm{B}}^{\mathrm{DTI}}$ and evaluated as follows:

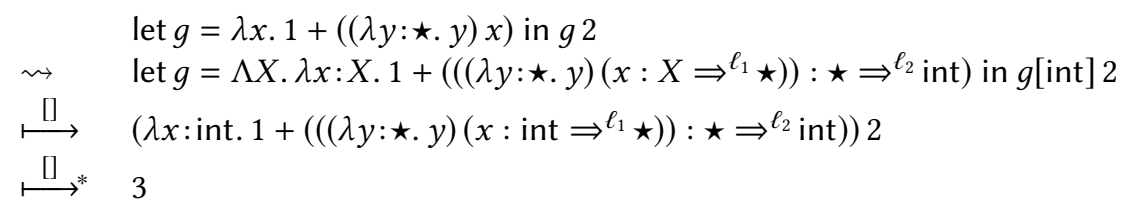

This program also evaluates to 3. Notice that $g$ is given a polymorphic type scheme $\forall X$. $X \rightarrow$ int because $x$ is passed to a function that expects an argument of the dynamic type and there is no constraint on its type. The use of $g$ comes with the type argument int so that it can be applied to an integer.

We show the evaluation sequence of the same term using the PBC-style semantics of type abstraction and application below:

$$
\begin{aligned}
& \text { let } g=\Lambda X \cdot \lambda x: X .1+\left(\left((\lambda y: \star \cdot y)\left(x: X \Rightarrow^{\ell_{1}} \star\right)\right): \star \Rightarrow^{\ell_{2}} \text { int }\right) \text { in } g[\text { int }] 2 \\
\stackrel{[]}{\longmapsto} & \left(\Lambda X \cdot \lambda x: X .1+\left(\left((\lambda y: \star \cdot y)\left(x: X \Rightarrow^{\ell_{1}} \star\right)\right): \star \Rightarrow^{\ell_{2}} \text { int }\right)\right)[\text { int }] 2 \\
\stackrel{[}{\longmapsto} & v X:=\text { int. }\left(\lambda x: X \cdot 1+\left(\left((\lambda y: \star \cdot y)\left(x: X \Rightarrow^{\ell_{1}} \star\right)\right): \star \Rightarrow^{\ell_{2}} \text { int }\right)\right) 2
\end{aligned}
$$

When a polymorphic function is applied to a type argument, a type binding $v X:=$ int is generated, instead of substituting int for $X$. As discussed by Ahmed et al. [2011, 2017], the generation of type bindings is crucial to ensure parametricity dynamically: $X$ behaves as if it is a fresh base type in the body of type abstraction, so evaluation proceeds as follows:

$$
\begin{aligned}
& \cdots \stackrel{[]}{\longmapsto} v X:=\text { int.1 }+\left(\left((\lambda y: \star . y)\left(2: X \Rightarrow^{\ell_{1}} \star\right)\right): \star \Rightarrow^{\ell_{2}} \text { int }\right) \\
& \stackrel{[]}{\longmapsto}^{*} \quad v X:=\text { int. } 1+\left(2: X \Rightarrow^{\ell_{1}} \star \Rightarrow^{\ell_{2}} \text { int }\right) \\
& \stackrel{[]}{\longmapsto} \text { blame } \ell_{2}
\end{aligned}
$$

The cast ( $2: X \Rightarrow^{\ell_{1}} \star \Rightarrow^{\ell_{2}}$ int) fails and this program evaluates to blame $\ell_{2}$, instead of 3 ! In fact, according to parametricity, the polymorphic type $\forall X . X \rightarrow$ int should behave the same regardless 
of $X$ and so it should uniformly return an integer constant or uniformly fail. From the viewpoint of parametricity, this is completely reasonable behavior.

In general, there is a conflict between polymorphism and blame in the PBC-based semantics. If $X$ were not generalized at let in the example above, $X$ would be unified with int and the resulting term

$$
\text { let } g=\lambda x: \text { int. } 1+\left(\left((\lambda y: \star \cdot y)\left(x: \text { int } \Rightarrow^{\ell_{1}} \star\right)\right): \star \Rightarrow^{\ell_{2}} \text { int }\right) \text { in } g 2
$$

would evaluate to 3 without blame. (This translation may be obtained by adapting the type inference algorithm to minimize the introduction of polymorphism [Bjørner 1994] to gradual typing.) The conflict can be considered another instance of incoherence in the sense that translations that differ in how let is generalized may result in different behavior.

We expect that our semantics is coherent and that operational equivalence between let $x=w$ in $f$ and $f[x:=w]$ holds (whereas it does not always hold in the PBC-based semantics as we saw above). However, they are achieved by sacrificing (dynamically enforced) parametricity.

\section{BASIC PROPERTIES OF $\lambda_{B}^{\text {DTI }}$}

In this section, we show basic properties of $\lambda_{B}^{\mathrm{DTI}}$ : type safety and conservativity over the standard blame calculus extended to let-polymorphism.

We first define the predicate $f \Uparrow$ to mean that $f$ diverges.

Definition 1 (Divergence). We write $f \Uparrow$ if there is an infinite evaluation sequence from $f$.

We write $f t v(f), f t v(U)$, and $f t v(\Gamma)$ for the set of free type variables in $f, U$, and $\Gamma$, respectively. We also write $\operatorname{dom}(S)$ for the domain of the type substitution $S$.

\subsection{Type Safety}

We show the type safety property using the progress and preservation lemmas [Wright and Felleisen 1994]. First, we state the canonical forms property below. As we have already mentioned, this lemma also means that no values are typed at type variables and this is why type variables are not ground types.

LEMma 3 (CANONiCAL Forms). If $\emptyset \vdash w: U$, then one of the following holds:

- $U=\iota$ and $w=c$ for some $\iota$ and $c$;

- $U=U_{1} \rightarrow U_{2}$ and $w=\lambda x: U_{1}$. f for some $x, f, U_{1}$, and $U_{2}$;

- $U=U_{1} \rightarrow U_{2}$ and $w=w^{\prime}: U_{1}^{\prime} \rightarrow U_{2}^{\prime} \Rightarrow^{\ell} U_{1} \rightarrow U_{2}$ for some $w^{\prime}, U_{1}, U_{2}, U_{1}^{\prime}, U_{2}^{\prime}$, and $\ell$; or

- $U=\star$ and $w=w^{\prime}: G \Rightarrow^{\ell} \star$ for some $w^{\prime}, G$, and $\ell$.

Proof. By case analysis on the last typing rule applied to derive $\emptyset \vdash w: U$.

The progress lemma is standard: A well-typed term can be evaluated one step further, or is a value or blame.

LEMma 4 (Progress). If $\emptyset \vdash f: U$, then one of the following holds: (1) $f \stackrel{S}{\longmapsto} f^{\prime}$ for some $S$ and $f^{\prime}$; (2) $f$ is a value; or (3) $f=$ blame $\ell$ for some $\ell$.

Proof. By induction on the typing derivation using Lemma 3.

The statement of the preservation lemma is slightly different from a standard one, because our semantics, which is equipped with DTI, may substitute type variables with some static type during reduction and evaluation.

Lemma 5 (Preservation). Suppose that $\emptyset \vdash f: U$. (1) If $\stackrel{S}{\longrightarrow} f^{\prime}$, then $\emptyset \vdash S\left(f^{\prime}\right): S(U)$ and (2) if $f \stackrel{S}{\longmapsto} f^{\prime}$, then $\emptyset \vdash f^{\prime}: S(U)$. 
Proof.

(1) By case analysis on the typing rule applied to $f$.

(2) By case analysis on the evaluation rule applied to $f$. In the case (E_STEP), use induction on the structure of $E$.

The second item (for the evaluation relation) does not require $S$ to be applied to $f^{\prime}$ in the type judgment (whereas the first item does) because it is already applied-see (E_STEP).

Finally, type safety holds in our blame calculus using the above lemmas. The following statement is also slightly different from a standard one in the literature for the same reason as the preservation.

THEOREM 1 (Type SAFETy). If $\emptyset \vdash f: U$, then one of the following holds: (1) $f \stackrel{S}{\longmapsto}$ * $r$ for some $S$ and $r$ such that $\emptyset \vdash r: S(U)$; or $(2) f \Uparrow$.

Proof. By Lemmas 4 and 5.

\subsection{Conservative Extension}

Our blame calculus is a conservative extension of the standard simply typed blame calculus [Siek et al. 2015a] extended to let-polymorphism (we call it $\lambda_{B}$ ). We can obtain $\lambda_{B}$ by disallowing free type variables and removing reduction rules (R_InStBASE) and (R_InSTARrow). We denote judgments and relations for this sublanguage by subscripting symbols with $\mathrm{B}$; we also omit type substitutions on the reduction/evaluation relations of $\lambda_{\mathrm{B}}$ because they are always empty. So, we write $f \longrightarrow_{\mathrm{B}} f^{\prime}$ for reduction and $f \longmapsto_{\mathrm{B}} f^{\prime}$ for evaluation. Then, it is easy to show that $\lambda_{\mathrm{B}}^{\mathrm{DTI}}$ is a conservative extension of $\lambda_{B}$ in the following sense.

Theorem 2 (Conservative Extension). Suppose that $f t v(f)=\emptyset$ and $f$ does not contain $v$.

(1) $f \stackrel{[]}{\longmapsto}^{*} r$ if and only iff $\longmapsto_{\mathrm{B}}^{*} r$.

(2) $f \Uparrow$ if and only iff $f \Uparrow_{\mathrm{B}}$.

\section{SOUNDNESS AND COMPLETENESS OF DYNAMIC TYPE INFERENCE}

In this section, we show soundness and completeness of DTI; we formalize these properties by comparing evaluation of a given term under $\lambda_{B}^{D T I}$ and its instances of type substitution in $\lambda_{B}$.

Soundness of static type inference means that, if the type inference algorithm succeeds, the program is well typed (under the reconstructed type annotation). In DTI, the success of type inference and the reconstructed type annotation roughly correspond to normally terminating evaluation and type substitution obtained through evaluation, respectively. So, soundness of DTI means that, if evaluation of a program normally terminates at value $w$ with a type substitution (yielded by DTI), then applying the type substitution before evaluation also makes evaluation normally terminate at the same value.

We state the soundness property below. In addition to normally terminating programs, we can show similar results for programs that abort by blame (the second item) or diverge (the third item). The second item means that, if a well-typed term evaluates to blame with DTI, then no substitution $S^{\prime}$ can help $S^{\prime}(f)$ avoid blame. In other words, DTI avoids blame as much as possible.

Theorem 3 (Soundness of Dynamic Type Inference). Suppose $\emptyset \vdash f: U$.

(1) If $\stackrel{S}{\longmapsto} *$, then, for any $S^{\prime}$ such that $f t v\left(S^{\prime}(S(f))\right)=\emptyset, S^{\prime}(S(f)) \stackrel{S^{\prime \prime}}{\longmapsto} S^{\prime}(r)$ for some $S^{\prime \prime}$.

(2) If $\stackrel{S}{\longmapsto}$ * blame $\ell$, then, for any $S^{\prime}, S^{\prime}(f) \stackrel{S^{\prime \prime}}{\longmapsto}$ * blame $\ell^{\prime}$ for some $S^{\prime \prime}$ and $\ell^{\prime}$.

(3) If $f \Uparrow$, then, for any $S$ such that $f t v(S(f))=\emptyset$, either $S(f) \Uparrow$ or $S(f) \stackrel{S^{\prime}}{\longmapsto}$ * blame $\ell$ for some $\ell$ and $S^{\prime}$. 
We state a main lemma to prove Theorem 3(1) below.

LEMMA 6. If $\stackrel{S_{1} \uplus S_{2}}{\longmapsto} * r$ and $\operatorname{dom}\left(S_{1}\right) \subseteq f t v(f)$, then $S_{1}(f) \stackrel{S_{2}^{\prime}}{\longmapsto} * r$ for some $S_{2}^{\prime}$ such that $\operatorname{dom}\left(S_{2}^{\prime}\right) \subseteq \operatorname{dom}\left(S_{2}\right)$ and $\operatorname{ftv}\left(S_{1}(X)\right) \cap \operatorname{dom}\left(S_{2}^{\prime}\right)=\emptyset$ for any $X \in \operatorname{dom}\left(S_{1}\right)$.

Here, $S_{1} \uplus S_{2}$ is a type substitution generated by DTI. ${ }^{7}$ Since fresh type variables are generated during reduction, we split the type substitution into two parts: $S_{1}$ for type variables that appear in $f$ and $S_{2}$ for generated type variables. The conditions on the type substitution $S_{2}^{\prime}$ mean that $S_{1}(f)$ may generate fewer type variables than $f$ and the domain of $S_{2}^{\prime}$ is fresh (with respect to $S_{1}(f)$ ). The statement is similar to Theorem 3(1) but additional conditions make proof by induction on the number of evaluation steps work.

We cannot say much about diverging programs-Theorem 3(3) means that, if a well-typed program diverges, then no type substitution $S$ makes $S(f)$ normally terminating. One may expect a stronger property that, if evaluation with DTI diverges, then without DTI it also diverges (after applying some type substitution that instantiates all type variables); but actually it is not the case.

THEOREM 4. There exists $f$ such that (1) $\emptyset \vdash f: U$ and (2) $f \Uparrow$ and (3) for any $S$ such that $f t v(S(f))=\emptyset$, it holds that $S(f) \longmapsto{ }_{\mathrm{B}}^{*}$ blame $\ell$ for some $\ell$.

Proof. Let $f=\left(\left(\lambda x: X .\left(x: X \Rightarrow^{\ell} \star^{\ell} \star \rightarrow \star\right)\left(x: X \Rightarrow^{\ell} \star\right)\right): X \rightarrow \star^{\ell} \star \rightarrow \star\right)((\lambda x: \star .(x:$ $\left.\left.\left.\star \Rightarrow^{\ell} \star \rightarrow \star\right) x\right): \star \rightarrow \star \Rightarrow^{\ell} \star\right)$. We can show that $\emptyset \vdash f: \star$ and $f \Uparrow$ and $S(f) \longmapsto_{\mathrm{B}}$ blame $\ell^{\prime}$ for any $S$ where $f t v(S(f))=\emptyset$.

The term $f$ in the proof is essentially the combinator $\Omega=(\lambda x, x x)(\lambda x, x x)$, for which we would need recursive types to give a type (if we did not use the dynamic type). Correspondingly, evaluation of $f$ diverges while generating type substitutions and fresh type variables infinitely often: $X$ is instantiated to $X_{1} \rightarrow X_{2}$ by (R_InstARrow), $X_{1}$ is instantiated to $X_{3} \rightarrow X_{4}, X_{3}$ is instantiated to $X_{5} \rightarrow X_{6}$, and so on. On the other hand, if we instantiate the type variable $X$ in $f$ with a finite static type without type variables, evaluation will eventually reach a cast $w: \star \rightarrow \star \Rightarrow^{\ell_{1}} \star \Rightarrow^{\ell_{2}} \iota$ and fail.

Completeness of ordinary static type inference means that, if there is some type substitution that makes a given program well typed, the type inference algorithm succeeds and finds a more general type substitution. A similar property holds for DTI: if there is some type substitution that makes a given program normally terminating, evaluation with DTI also results in a related value and the obtained type substitution is more general. We can also prove that, if there is some type substitution that makes a program diverge, then evaluation with DTI also diverges.

The main lemma to prove completeness is below. It intuitively means completeness for one step: if a term $S(f)$ evaluates in one step and it does not result in blame, then the original, uninstantiated term $f$ also evaluates with DTI. The assumption "it does not result in blame", which means that the first type substitution is a good one, is crucial.

LEMma 7. If $\emptyset \vdash f: U$ and $S(f) \stackrel{S_{1}^{\prime}}{\longmapsto} f^{\prime}$ and $f^{\prime} \stackrel{S_{\rho}}{\longmapsto} *$ blame $\ell$ for any $S_{0}$ and $\ell$, then $f \stackrel{S^{\prime}}{\longmapsto} f^{\prime \prime}$ and $S^{\prime \prime}\left(f^{\prime \prime}\right)=f^{\prime}$ for some $S^{\prime}, S^{\prime \prime}$, and $f^{\prime \prime}$.

Proof. By case analysis on the evaluation rule applied to $S(f)$.

We state the completeness property of DTI as follows:

Theorem 5 (Completeness of Dynamic Type Inference). Suppose $\emptyset$ $f: U$.

(1) If $S(f) \stackrel{S^{\prime}}{\longmapsto} *$, then $f \stackrel{S^{\prime \prime}}{\longmapsto} * w^{\prime}$ and $S^{\prime \prime \prime}\left(w^{\prime}\right)=w$ for some $w^{\prime}, S^{\prime \prime}$, and $S^{\prime \prime \prime}$.

\footnotetext{
${ }^{7}$ The concatenation $S_{1} \uplus S_{2}$ of $S_{1}$ and $S_{2}$ is defined only if $\operatorname{dom}\left(S_{1}\right)$ and $\operatorname{dom}\left(S_{2}\right)$ are disjoint and it maps $X$ to $S_{1}(X)$ if $X \in \operatorname{dom}\left(S_{1}\right)$ and to $S_{2}(X)$ if $X \in \operatorname{dom}\left(S_{2}\right)$.
} 
(2) If $S(f) \Uparrow$, then $f \Uparrow$.

Proof. By Lemma 7.

In the first item, $S^{\prime \prime}$ is required to relate the two values because DTI may still leave some type variables uninstantiated. In fact, $S^{\prime \prime}$ witnesses that $S^{\prime}$ is more general than $S$.

Remark: As we discussed in Section 1.3, an alternative to DTI, which is to substitute $\star$ for type variables that type inference left uninstantiated, breaks the following property, which could be viewed as soundness:

If $\emptyset \vdash f: U, S$ substitutes $\star$ for all type variables in $f$, and $S(f) \longmapsto{ }_{\mathrm{B}}^{*} w$, then there is some static type substitution $S^{\prime}$ such that $S^{\prime}(f) \longmapsto_{\mathrm{B}}^{*} w^{\prime}$ for some $w^{\prime}$.

The term

$$
\begin{aligned}
& \left(\lambda x: \star .\left(\left(\left(x: \star \Rightarrow^{\ell_{1}} \star \rightarrow \star\right)\left(2: \text { int } \Rightarrow^{\ell_{2}} \star\right)\right): \star \Rightarrow^{\ell_{3}} \star \rightarrow \star\right)\left(\text { true : bool } \Rightarrow^{\ell_{4}} \star\right)\right) \\
& \quad\left(\left(\lambda y_{1}: Y . \lambda y_{2}: Y \text {. if } b \text { then } y_{1} \text { else } y_{2}\right): \star \rightarrow \star \rightarrow \star \Rightarrow^{\ell_{5}} \star\right)
\end{aligned}
$$

is a counterexample. However, completeness would be satisfied. The semantics where uninstantiated type variables are regarded as distinguished base types, as in Garcia and Cimini [2015], would satisfy neither the second item of soundness nor completeness.

\section{THE GRADUAL GUARANTEE}

The gradual guarantee [Siek et al. 2015a], a property capturing the essence of gradual code evolution, is one of the important criteria for gradually typed languages. It formalizes the intuition that, in a gradual type system, giving more "precise" type annotations can find more typing errors either statically or dynamically but otherwise does not change the behavior of programs. In a simple setting, a type is more precise than another, if the former is obtained by replacing some occurrences of $\star$ by other types. For example, int $\rightarrow \star \rightarrow$ bool is more precise than $\star \rightarrow \star$. The statement consists of two parts: one about typeability (the static gradual guarantee) and the other about evaluation (the dynamic gradual guarantee).

The static gradual guarantee states that, if term $e$ is well typed, then so is a term that is the same as $e$ except that it is less precisely annotated.

The dynamic gradual guarantee ensures that a more precisely annotated program and a less precisely annotated one behave in the same way as far as the more precisely annotated program does not raise blame. For example, let us consider the following gradually typed program (which is similar to the example used in Section 1):

$$
(\lambda x: \star \rightarrow \star . x 2)(\lambda y: \star \cdot y)
$$

This program is translated into a term in the blame calculus and evaluates to value $2:$ int $\Rightarrow^{\ell} \star$. On one hand, we can obtain a less precisely annotated program by replacing the type annotation $\star \rightarrow \star$ for variable $x$ with $\star$, which is the least precise type.

$$
(\lambda x: \star . x 2)(\lambda y: \star . y)
$$

This program evaluates to the same value $2:$ int $\Rightarrow^{\ell} \star$ as the gradual guarantees expects. On the other hand, we can get a more precisely annotated program by replacing the type annotation $\star$ for variable $y$ with int.

$$
(\lambda x: \star \rightarrow \star . x 2)(\lambda y: \text { int. } y)
$$

Then, it also evaluates to the same value 2 : int $\Rightarrow^{\ell} \star$. However, more precisely annotated programs may raise blame even if less precisely annotated ones do not. For example, let us consider the case 
that $y$ in the above program is given a wrong type annotation, e.g., bool.

$$
(\lambda x: \star \rightarrow \star . x 2)(\lambda y: \text { bool. } y)
$$

Then, it raises blame because the evaluation triggers a sequence of casts 2 : int $\Rightarrow^{\ell_{1}} \star \Rightarrow^{\ell_{2}}$ bool, which fails.

In the rest of this section, we show that the ITGL satisfies both the static and dynamic gradual guarantee. We extend the statements to take static/dynamic type inference into account: For example, the statement of the static gradual guarantee becomes that, given $e$, if static type inference succeeds, then for any less precisely annotated term $e^{\prime}$ static type inference also succeeds. To state formally, we first introduce the ITGL and translation of ITGL terms to $\lambda_{\mathrm{B}}^{\mathrm{DTI}}$ terms. Then, the notion of "more (or less) precisely annotated" ITGL terms is formalized by a precision relation over the ITGL terms. Using the precision, we show the static gradual guarantee. The dynamic gradual guarantee is proved by giving a precision relation also for $\lambda_{\mathrm{B}}^{\mathrm{DTI}}$ and showing the two properties: (1) the translation results of precision-related ITGL terms are precision-related; and (2) precision-related $\lambda_{\mathrm{B}}^{\mathrm{DTI}}$ terms behave equivalently if the more precisely annotated term does not raise blame.

\subsection{ITGL: The Implicitly Typed Gradual Language}

5.1.1 Definition. This section reviews the definition of the ITGL [Garcia and Cimini 2015]. Figure 5 shows the syntax and the typing rules of the ITGL and the cast insertion rules to translate the ITGL to $\lambda_{\mathrm{B}}^{\mathrm{DTI}}$. The syntax is from the standard lambda calculus, except that it provides two kinds of abstractions: one is $\lambda x: U$.e, where the type of argument $x$ is given as $U$ explicitly, and the other is $\lambda x$. $e$, where the type of $x$ is implicit. A let-expression let $x=v$ in $e$ allows $v$ to be polymorphic in $e$; type variables to be generalized are implicit in terms of the ITGL, while they are explicit in $\lambda_{\mathrm{B}}^{\mathrm{DTI}}$.

The typing rules are essentially the same as what is called schematic typing in Garcia and Cimini [2015] modulo a few minor adaptations. The rules (IT_VARP) and (IT_ABsI) require implicit types to be static. The rule (IT_Op) says that types of arguments have to be consistent with the argument types of op. The rule (IT_App) for application $e_{1} e_{2}$ is standard in gradual typing [Siek et al. 2015a]. The type $U_{1}$ of $e_{1}$ is consistent with function type $U_{11} \rightarrow U_{12}$ obtained by operation $\triangleright$, which is defined as

$$
\star \quad \star \quad U_{1} \rightarrow U_{2} \quad \triangleright \quad U_{1} \rightarrow U_{2},
$$

and the type $U_{2}$ of $e_{2}$ has to be consistent with the argument type of $U_{11} \rightarrow U_{12}$. The rule (IT_LET) is standard in languages with let-polymorphism [Milner 1978] except the condition on type variables to be generalized. We do not allow type variables that appear in type annotations of $v$ to be generalized because allowing it invalidates the property that let $x=v$ in $e$ is well typed if and only if so is $e[x:=v]$ (if $e$ refers to $x$ ). For example, let us consider ITGL program

$$
\text { let } x=\lambda y: X . y \text { in }(x 2, x \text { true }) \text {. }
$$

This program would be well typed if $X$ could be generalized. However, the result of expanding let

$$
(x 2, x \text { true })[x:=\lambda y: X . y]=((\lambda y: X . y) 2,(\lambda y: X \cdot y) \text { true })
$$

is not well typed because $X$ could not be instantiated in two ways: int and bool. Our restriction to generalizable type variables rejects not only the latter but also the former; OCaml seems to adopt the same strategy (such type variables are generalized only at the top level).

Translation $\Gamma \vdash e \rightsquigarrow f: U$ of ITGL term $e$ of type $U$ to $\lambda_{\mathrm{B}}^{\mathrm{DTI}}$ term $f$ is achieved by inserting casts and making implicit type information explicit. The cast insertion rules, which are shown in the lower half of Figure 5, are standard [Siek et al. 2015a] except (CI_VARP) and (CI_LETP). The rule (CI_VARP) makes type instantiations explicit in terms. The side condition is the same as that of 
Syntax:

$$
\begin{array}{ll}
\text { Terms } & e::=x|c| o p(e, e)|\lambda x: U . e| \lambda x . e|e e| \text { let } x=v \text { in } e \\
\text { Values } & v::=c|\lambda x: U . e| \lambda x . e
\end{array}
$$

Typing rules: $\Gamma+e: U$

$$
\begin{gathered}
\frac{x: \forall \vec{X}_{i} \cdot U \in \Gamma}{\Gamma \vdash x: U\left[\vec{X}_{i}:=\vec{T}_{i}\right]} \text { IT_VARP } \\
\frac{t y(o p)=\iota_{1} \rightarrow \iota_{2} \rightarrow \iota \quad \Gamma \vdash e_{1}: U_{1} \quad \Gamma \vdash e_{2}: U_{2} \quad U_{1} \sim \iota_{1} \quad U_{2} \sim \iota_{2}}{\Gamma \vdash o p\left(e_{1}, e_{2}\right): \iota} \text { IT_OP } \\
\frac{\Gamma, x: U_{1} \vdash e: U_{2}}{\Gamma \vdash \lambda x: U_{1} \cdot e: U_{1} \rightarrow U_{2}} \text { IT_ABsE } \quad \frac{\Gamma, x: T \vdash e: U}{\Gamma \vdash \lambda x . e: T \rightarrow U} \text { IT_ABsI } \\
\frac{\Gamma \vdash e_{1}: U_{1} \quad \Gamma \vdash e_{2}: U_{2} \quad U_{1} \triangleright U_{11} \rightarrow U_{12} \quad U_{2} \sim U_{11}}{\Gamma \vdash e_{1} e_{2}: U_{12}} \text { IT_APP } \\
\frac{\Gamma \vdash v_{1}: U_{1} \quad \Gamma, x: \forall \vec{X}_{i} U_{1} \vdash e_{2}: U_{2} \quad \vec{X}_{i}=f t v\left(U_{1}\right) \backslash\left(f t v(\Gamma) \cup f t v\left(v_{1}\right)\right)}{\Gamma \vdash \operatorname{let} x=v_{1} \text { in } e_{2}: U_{2}} \text { IT_LETP }
\end{gathered}
$$

Cast insertion rules: $\Gamma \vdash e \rightsquigarrow f: U$

$$
\begin{aligned}
& \frac{x: \forall \vec{X}_{i} \cdot U \in \Gamma \quad \text { For any } X_{j} \in \vec{X}_{i}, \mathbb{T}_{j}=v \text { iff } X_{j} \notin f t v(U)}{\Gamma \vdash x \rightsquigarrow x\left[\overrightarrow{\mathbb{T}}_{i}\right]: U\left[\vec{X}_{i}:=\overrightarrow{\mathbb{T}}_{i}\right]} \text { CI_VARP } \\
& \Gamma \vdash e_{1} \rightsquigarrow f_{1}: U_{1} \quad \Gamma \vdash e_{2} \rightsquigarrow f_{2}: U_{2} \\
& \frac{t y(o p)=\iota_{1} \rightarrow \iota_{2} \rightarrow \iota \quad U_{1} \sim \iota_{1} \quad U_{2} \sim \iota_{2}}{\Gamma \vdash c \rightsquigarrow c: t y(c)} \text { CI_CONST } \quad \frac{{ }_{1}}{\Gamma \vdash o p\left(e_{1}, e_{2}\right) \rightsquigarrow o p\left(f_{1}: U_{1} \Rightarrow^{\ell_{1}} \iota_{1}, f_{2}: U_{2} \Rightarrow^{\ell_{2}} \iota_{2}\right): \iota} \text { CI_OP } \\
& \frac{\Gamma, x: T \vdash e \rightsquigarrow f: U}{\Gamma \vdash \lambda x . e \rightsquigarrow \lambda x: T . f: T \rightarrow U} \text { CI_AbsI } \frac{\Gamma, x: U_{1} \vdash e \rightsquigarrow f: U_{2}}{\Gamma \vdash \lambda x: U_{1} . e \rightsquigarrow \lambda x: U_{1} \cdot f: U_{1} \rightarrow U_{2}} \text { CI_ABsE } \\
& \frac{\Gamma \vdash e_{1} \rightsquigarrow f_{1}: U_{1} \quad \Gamma \vdash e_{2} \rightsquigarrow f_{2}: U_{2} \quad U_{1} \triangleright U_{11} \rightarrow U_{12} \quad U_{2} \sim U_{11}}{\Gamma \vdash e_{1} e_{2} \rightsquigarrow\left(f_{1}: U_{1} \Rightarrow^{\ell_{1}} U_{11} \rightarrow U_{12}\right)\left(f_{2}: U_{2} \Rightarrow^{\ell_{2}} U_{11}\right): U_{12}} \text { CI_APP } \\
& \Gamma \vdash v_{1} \rightsquigarrow w_{1}: U_{1} \quad \Gamma, x: \forall \vec{X}_{i} \vec{Y}_{j} \cdot U_{1} \vdash e_{2} \rightsquigarrow f_{2}: U_{2} \\
& \frac{\vec{X}_{i}=f t v\left(U_{1}\right) \backslash\left(f t v(\Gamma) \cup f t v\left(v_{1}\right)\right)}{\Gamma \vdash \text { let } x=v_{1} \text { in } e_{2} \rightsquigarrow \text { let } x=\Lambda{\overrightarrow{X_{i}}}_{j} \cdot w_{1} \text { in } f_{2}: U_{2}} \text { CI_LETP }
\end{aligned}
$$

Fig. 5. The ITGL.

(T_VARP). The rule (CI_LETP) is similar to (T_LETP), but it allows not only type variables $\vec{X}_{i}$ that appear in the type $U_{1}$ of polymorphic value $w_{1}$ but also $\vec{Y}_{i}$ that appear only in $w_{1}$-they are implicit in ITGL terms - to be generalized for identifying let $x=v$ in $e$ with $e[x:=v]$ semantically. For example, let us consider the following ITGL term:

$$
\text { let } x=\lambda y \cdot((\lambda z \cdot z):: \star \rightarrow \star) y \text { in }(x 2, x \text { true })
$$


where $e:: U$ means $(\lambda x: U . x)$ e. If type variables that appear in only $U_{1}$ could be generalized, it would be translated to

$$
\text { let } x=\Lambda X . \lambda y: X .\left((\lambda z: Y . z): Y \rightarrow Y \Rightarrow^{\ell_{1}} \star \rightarrow \star\right)\left(y: X \Rightarrow^{\ell_{2}} \star\right) \text { in }(x[\text { int }] 2, x[\text { bool }] \text { true })
$$

where $Y$ is not generalized. The evaluation of this term results in blame because the monomorphic type variable $Y$ will be instantiated with different base types int and bool by DTI. However, the evaluation based on let-expansion does not trigger blame:

$$
\begin{aligned}
& (x 2, x \text { true })[x:=\lambda y \cdot((\lambda z \cdot z):: \star \rightarrow \star) y] \\
& =\quad((\lambda y \cdot((\lambda z \cdot z):: \star \rightarrow \star) y) 2,(\lambda y \cdot((\lambda z \cdot z):: \star \rightarrow \star) y) \text { true }) \\
& \rightsquigarrow \quad\left(\left(\lambda y: \text { int. }\left(\left(\lambda z: Y_{1} . z\right): Y_{1} \rightarrow Y_{1} \Rightarrow^{\ell_{1}} \star \rightarrow \star\right)\left(y: \text { int } \Rightarrow^{\ell_{2}} \star\right)\right) 2\right. \\
& \left.\left(\lambda y: \text { bool. }\left(\left(\lambda z: Y_{2} . z\right): Y_{2} \rightarrow Y_{2} \Rightarrow^{\ell_{3}} \star \rightarrow \star\right)\left(y: \text { bool } \Rightarrow^{\ell_{4}} \star\right)\right) \text { true }\right)^{8} \\
& \stackrel{\left[Y_{1}:=\mathrm{int}\right]}{\longrightarrow} *\left(\left(2: \text { int } \Rightarrow^{\ell_{1}} \star\right),\left(\lambda y: \text { bool. }\left(\left(\lambda z: Y_{2} \cdot z\right): Y_{2} \rightarrow Y_{2} \Rightarrow^{\ell_{3}} \star \rightarrow \star\right)\left(y: \text { bool } \Rightarrow^{\ell_{4}} \star\right)\right) \text { true }\right) \\
& \stackrel{\left[Y_{2}:=\text { bool }\right]}{\longmapsto} *\left(\left(2: \text { int } \Rightarrow^{\ell_{1}} \star\right),\left(\text { true }: \text { bool } \Rightarrow^{\ell_{3}} \star\right)\right)
\end{aligned}
$$

By allowing generalization of type variables appearing in not only $U_{1}$ but also $w_{1}$ (but not $v_{1}$ ), we achieve the same semantics as let-expansion.

We show that the cast insertion is type-preserving and type safety of the ITGL.

Theorem 6 (CAst Insertion is Type-Preserving). If $\Gamma \vdash e: U$, then $\Gamma \vdash e \rightsquigarrow f: U$ and $\Gamma \vdash f: U$ for some $f$.

Definition 2 (Evaluation of ITGL terms). We write $\langle\Gamma \vdash e: U\rangle \stackrel{S}{\longmapsto}{ }^{*} f$ if $\Gamma \vdash e \rightsquigarrow f^{\prime}: U$ and $f^{\prime} \stackrel{S}{\longmapsto}{ }^{*} f$ for some $f^{\prime}$. We also write $\langle\Gamma \vdash e: U\rangle \Uparrow$ if $\Gamma \vdash e \rightsquigarrow f: U$ and $f \Uparrow$ for some $f$.

Corollary 1 (Type SAfety of THE ITGL). If $\emptyset$ - $e: U$, then:

- $\langle\emptyset \vdash e: U\rangle \stackrel{S}{\longmapsto} * w$ for some $S$ and $w$ such that $\emptyset \vdash w: S(U)$;

- $\langle\emptyset \vdash e: U\rangle \stackrel{S}{\longrightarrow}$ blame $\ell$ for some $S$ and $\ell$; or

- $\langle\emptyset+e: U\rangle \Uparrow$.

5.1.2 Precision. We introduce precision relations for the ITGL to formalize more precise types and more precisely annotated terms.

Type precision. As we have already mentioned, intuitively, type $U$ is more precise than type $U^{\prime}$ if $U$ is obtained by replacing some occurrences of the dynamic type in $U^{\prime}$ with other types. We generalize this notion of precision to type variables. Type variables are similar to the dynamic type in that type variables that appear in well-typed terms can be instantiated with any type as values of any type can flow to the dynamic type. However, they differ from the dynamic type in that all occurrences of a type variable have to be instantiated with a single static type. For example, int $\rightarrow$ bool can be considered-and is in our precision-more precise than $X$ because we get the former by instantiating $X$ with int $\rightarrow$ bool, while it cannot be compared with $X \rightarrow X$ because $X$ is not allowed to be instantiated in two different ways: int and bool.

To ensure that all occurrences of a type variable are instantiated in the same way, we equip type precision $U \sqsubseteq_{S} U^{\prime}$, which means that $U$ is more precise than $U^{\prime}$, with type substitution $S$, which gives instantiations of type variables in $U^{\prime}$. The type precision rules, given in the top of Figure 6, are standard [Siek et al. 2015a] except (P_TYVAR): a base type is more precise than itself (P_IDBASE); components of precision-related function types are also precision-related (P_ARROw); and the dynamic type is the least precise type (P_Dyn). The rule (P_TrVAR) allows type variables

\footnotetext{
${ }^{8} \mathrm{We}$ omit trivial identity functions inserted due to type ascription here.
} 


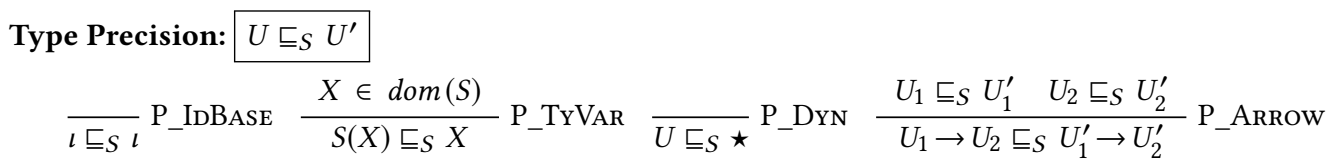

$$
\begin{aligned}
& \text { Untyped Term Precision: } e \sqsubseteq S e^{\prime}
\end{aligned}
$$

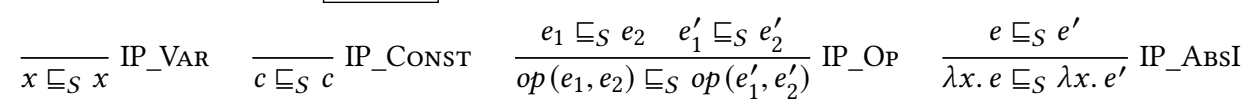

$$
\begin{aligned}
& \frac{e \sqsubseteq_{S} e^{\prime}}{\lambda x . e \sqsubseteq_{S} \lambda x: \star \cdot e^{\prime}} \text { IP_ABsIE } \frac{e \sqsubseteq_{S} e^{\prime}}{\lambda x: T_{1} \cdot e \sqsubseteq_{S} \lambda x \cdot e^{\prime}} \text { IP_ABsEI } \frac{U \sqsubseteq_{S} U^{\prime} \quad e \sqsubseteq_{S} e^{\prime}}{\lambda x: U . e \sqsubseteq_{S} \lambda x: U^{\prime} \cdot e^{\prime}} \text { IP_AbsE } \\
& \frac{e_{1} \sqsubseteq_{S} e_{1}^{\prime} \quad e_{2} \sqsubseteq_{S} e_{2}^{\prime}}{e_{1} e_{2} \sqsubseteq_{S} e_{1}^{\prime} e_{2}^{\prime}} \text { IP_APP } \frac{v_{1} \sqsubseteq_{S} v_{1}^{\prime} \quad e_{2} \sqsubseteq_{S} e_{2}^{\prime}}{\operatorname{let} x=v_{1} \text { in } e_{2} \sqsubseteq_{S} \text { let } x=v_{1}^{\prime} \text { in } e_{2}^{\prime}} \text { IP_LETP }
\end{aligned}
$$

Fig. 6. Precision of the ITGL.

to be instantiated according to $S$. If we want to relate $X$ to itself, we can give substitution $[X:=X]$. This type precision cooperates well with DTI in the sense that, if the evaluation of a more precisely annotated term using type annotation int $\rightarrow$ bool does not raise blame, then that of a less precisely annotated one using $X$ does not, either, because DTI would instantiate $X$ with int $\rightarrow$ bool or a less precise static type (such as $Y \rightarrow$ bool).

Term precision. Rules of term precision $e \sqsubseteq_{S} e^{\prime}$, which means that $e$ is more precisely annotated than $e^{\prime}$, are given in the bottom of Figure 6. All rules but (IP_ABsIE), (IP_ABsEI), and (IP_ABsE) are just for compatibility. Lambda abstraction $\lambda x . e$ is more precisely annotated than $\lambda x: \star . e$ (IP_ABsIE) because the static type inference gives variable $x$ in $\lambda x . e$ a static type (if any), which is more precise than the dynamic type. Lambda abstraction $\lambda x:$ T.e is more precisely annotated than $\lambda x . e$ (IP_ABsEI) because the static type inference algorithm by Garcia and Cimini [2015] gives a principal static type to $x$ in $\lambda x$. $e$ and it should be less precise than $T$ under some type substitution due to principality. Finally, $\lambda x: U . e$ is more precisely annotated than $\lambda x: U^{\prime} . e^{\prime}$ if $U$ is more precise than $U^{\prime}$ and $e$ is more precisely annotated than $e^{\prime}$ under $S$ (IP_ABsE).

\subsection{The Static Gradual Guarantee}

Definition 3 (Principal Type Inference). We suppose that there is a partial function $P T(\Gamma, e)$ that (1) if $S^{\prime}(\Gamma) \vdash S^{\prime}(e): U^{\prime}$ for some $S^{\prime}$ and $U^{\prime}$, produces a pair $(S, U)$ such that (a) $S(\Gamma) \vdash S(e): U$ and (b) for any $S_{1}$ and $U_{1}$ such that $S_{1}(\Gamma) \vdash S_{1}(e): U_{1}$, there exists $S_{2}$ such that $S_{1}=S_{2} \circ S$; and (2) is undefined otherwise.

We can obtain a principal type inference algorithm PT from Garcia and Cimini [2015]. Now, we show the static gradual guarantee for the ITGL.

Theorem 7 (Static Gradual Guarantee). If $e \sqsubseteq S_{0} e^{\prime}$ and PT $(\emptyset, e)=\left(S_{1}, U\right)$, then PT $\left(\emptyset, e^{\prime}\right)=$ $\left(S_{1}^{\prime}, U^{\prime}\right)$ and $U \sqsubseteq_{S_{2}} U^{\prime}$ for some $S_{2}, S_{1}^{\prime}$, and $U^{\prime}$.

\subsection{Precision of $\lambda_{\mathbf{B}}^{\text {DTI }}$}

As described at the beginning of this section, we show the dynamic gradual guarantee via reduction of ITGL term precision to $\lambda_{\mathrm{B}}^{\mathrm{DTI}}$ term precision. We denote $\lambda_{\mathrm{B}}^{\mathrm{DTI}}$ term precision by

$$
\left\langle\Gamma \vdash f: U \sqsubseteq S U^{\prime}: f^{\prime} \dashv \Gamma^{\prime}\right\rangle,
$$



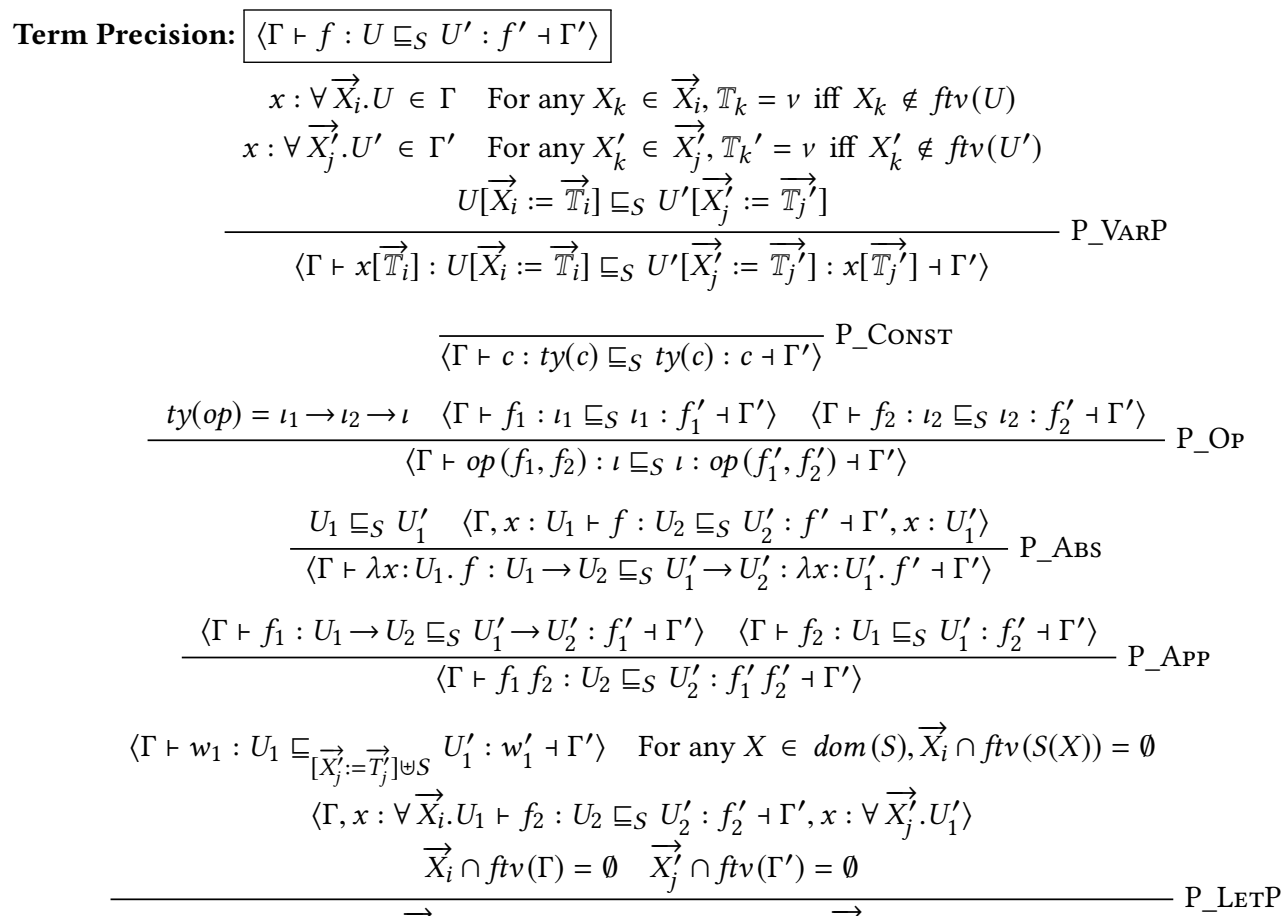

$\left\langle\Gamma\right.$ เ let $x=\Lambda \vec{X}_{i} \cdot w_{1}$ in $f_{2}: U_{2} \sqsubseteq_{S} U_{2}^{\prime}:$ let $x=\Lambda \vec{X}_{j}^{\prime} \cdot w_{1}^{\prime}$ in $\left.f_{2}^{\prime} \dashv \Gamma^{\prime}\right\rangle$

$$
\begin{gathered}
\frac{\left\langle\Gamma \vdash f: U_{1} \sqsubseteq_{S} U_{1}^{\prime}: f^{\prime} \dashv \Gamma^{\prime}\right\rangle \quad U_{1} \sim U_{2} \quad U_{1}^{\prime} \sim U_{2}^{\prime} \quad U_{2} \sqsubseteq_{S} U_{2}^{\prime}}{\left\langle\Gamma \vdash\left(f: U_{1} \Rightarrow^{\ell} U_{2}\right): U_{2} \sqsubseteq_{S} U_{2}^{\prime}:\left(f^{\prime}: U_{1}^{\prime} \Rightarrow^{\ell^{\prime}} U_{2}^{\prime}\right) \dashv \Gamma^{\prime}\right\rangle} \text { P_CAST } \\
\frac{\left\langle\Gamma \vdash f: U_{1} \sqsubseteq_{S} U^{\prime}: f^{\prime} \dashv \Gamma^{\prime}\right\rangle \quad U_{1} \sim U \quad U \sqsubseteq_{S} U^{\prime}}{\left\langle\Gamma \vdash\left(f: U_{1} \Rightarrow^{\ell} U\right): U \sqsubseteq_{S} U^{\prime}: f^{\prime} \dashv \Gamma^{\prime}\right\rangle} \text { P_CAsTL } \\
\frac{\left\langle\Gamma \vdash f: U \sqsubseteq_{S} U_{1}^{\prime}: f^{\prime} \dashv \Gamma^{\prime}\right\rangle \quad U_{1}^{\prime} \sim U^{\prime} \quad U \sqsubseteq_{S} U^{\prime}}{\left\langle\Gamma \vdash f: U \sqsubseteq_{S} U^{\prime}:\left(f^{\prime}: U_{1}^{\prime} \Rightarrow^{\ell^{\prime}} U^{\prime}\right) \dashv \Gamma^{\prime}\right\rangle} \text { P_CASTR } \\
\frac{\Gamma^{\prime} \vdash f^{\prime}: U^{\prime} U \sqsubseteq_{S} U^{\prime}}{\left\langle\Gamma \vdash \text { blame } \ell: U \sqsubseteq_{S} U^{\prime}: f^{\prime} \dashv \Gamma^{\prime}\right\rangle} \text { P_BLAme }
\end{gathered}
$$

Fig. 7. Term precision of $\lambda_{\mathrm{B}}^{\mathrm{DTI}}$.

which means that $\lambda_{\mathrm{B}}^{\mathrm{DTI}}$ term $f$ having type $U$ under $\Gamma$ is more precisely annotated than $f^{\prime}$ having type $U^{\prime}$ under $\Gamma^{\prime}$; type substitution $S$ plays the same role as in precision of the ITGL.

We show precision rules of $\lambda_{\mathrm{B}}^{\mathrm{DTI}}$ in Figure 7. The rules (P_VAR), (P_Const), (P_Op), (P_ABs), (P_APP), and (P_CAST) are similar to the corresponding typing rules of $\lambda_{\mathrm{B}}^{\mathrm{DTI}}$ except that they ensure that type information on the left-hand side is more precise than that on the right-hand side. The rule (P_LETP) relates two let-expressions let $x=\Lambda \vec{X}_{i} \cdot w_{1}$ in $f_{2}$ and let $x=\Lambda \overrightarrow{X_{j}^{\prime}} \cdot w_{1}^{\prime}$ in $f_{2}^{\prime}$. This rule allows polymorphic values $w_{1}$ and $w_{1}^{\prime}$ to be related under $S$ augmented with a type substitution $\left[\overrightarrow{X_{j}^{\prime}}:=\overrightarrow{T_{j}^{\prime}}\right]$, which maps bound type variables $\overrightarrow{X_{j}^{\prime}}$ on the imprecise side to more precise types $\overrightarrow{T_{j}^{\prime}}$ on the precise side. The second premise claims that $S$ does not capture bound variables $\vec{X}_{i}$; note that free type variables in types to which $S$ maps may appear on only the precise side. The rules 
(P_CASTL) and (P_CASTR) are given because modifying type annotations of casts changes casts generated at run time. The rule (P_BLAME) represents that a term on the precise side may involve blame even if one on the imprecise side does not.

\subsection{The Dynamic Gradual Guarantee}

Now, we show the dynamic gradual guarantee.

Theorem 8 (Dynamic Gradual Guarantee). Suppose that $e \sqsubseteq S_{0} e^{\prime}$. Let $(S, U)=P T(\emptyset, e)$ and $\left(S^{\prime}, U^{\prime}\right)=P T\left(\emptyset, e^{\prime}\right)$.

(1) • If $\langle\emptyset \vdash S(e): U\rangle \stackrel{S_{1}}{\longmapsto} *$, then $\left\langle\emptyset \vdash S^{\prime}\left(e^{\prime}\right): U^{\prime}\right\rangle \stackrel{S_{1}^{\prime}}{\longmapsto}{ }^{*} w^{\prime}$ and $\left\langle\emptyset \vdash w: S_{1}(U) \sqsubseteq S_{0}^{\prime} S_{1}^{\prime}\left(U^{\prime}\right)\right.$ : $\left.w^{\prime} \dashv \emptyset\right\rangle$ for some $w^{\prime}, S_{1}^{\prime}$, and $S_{0}^{\prime}$.

- If $\langle\emptyset \vdash S(e): U\rangle \Uparrow$, then $\left\langle\emptyset \vdash S^{\prime}\left(e^{\prime}\right): U^{\prime}\right\rangle \Uparrow$.

(2) • If $\left\langle\emptyset \vdash S^{\prime}\left(e^{\prime}\right): U^{\prime}\right\rangle \stackrel{S_{1}^{\prime}}{\longmapsto}{ }^{*} w^{\prime}$, then either $(1)\langle\emptyset \vdash S(e): U\rangle \stackrel{S_{1}}{\longmapsto} *$ and $\left\langle\emptyset \vdash w: S_{1}(U) \sqsubseteq S_{0}^{\prime}\right.$ $\left.S_{1}^{\prime}\left(U^{\prime}\right): w^{\prime} \dashv \emptyset\right\rangle$ for some $w, S_{1}$, and $S_{0}^{\prime}$; or $(2)\langle\emptyset \vdash S(e): U\rangle \stackrel{S_{1}}{\longmapsto}{ }^{*}$ blame $\ell$ for some $\ell$ and $S_{1}$.

- If $\left\langle\emptyset \vdash S^{\prime}\left(e^{\prime}\right): U^{\prime}\right\rangle \stackrel{S_{1}^{\prime}}{\longmapsto} *$ blame $\ell^{\prime}$, then $\langle\emptyset \vdash S(e): U\rangle \stackrel{S_{1}}{\longmapsto} *$ blame $\ell$ for some $\ell$ and $S_{1}$.

- If $\left\langle\emptyset \vdash S^{\prime}\left(e^{\prime}\right): U^{\prime}\right\rangle \Uparrow$, then either (1) $\langle\emptyset \vdash S(e): U\rangle \Uparrow$, or $(2)\langle\emptyset \vdash S(e): U\rangle \stackrel{S_{1}}{\longmapsto}$ * blame $\ell$ for some $\ell$ and $S_{1}$.

Proof. It is shown from auxiliary theorems that (1) the cast-inserting translation is precisionpreserving and (2) precision-related $\lambda_{\mathrm{B}}^{\mathrm{DTI}}$ terms behave equivalently if the precisely annotated term does not raise blame.

Connection between the gradual guarantee and the soundness-completeness of DTI. The dynamic gradual guarantee states that precise type annotations may find more type errors at run time but otherwise do not change the behavior of programs. Perhaps interestingly, the soundness and completeness of DTI also ensure a very similar property (although it is a property of the intermediate language, not the ITGL): they state that applying a type substitution may find more type errors at run time but otherwise does not change the behavior of programs. Roughly speaking, the completeness corresponds to the first item of the dynamic gradual guarantee and the soundness to the second item. Given the fact that $e=S\left(e^{\prime}\right)$ implies $e \sqsubseteq_{S} e^{\prime}$, the premise of Theorem 8 is weaker than those of Theorems 3 and 5. However, the conclusions of the soundness and completeness are stronger than that of the gradual guarantee: if compared terms evaluate to values, they are syntactically equivalent modulo type instantiations, while in the gradual guarantee the evaluation results may have different occurrences of the dynamic type. Moreover, Theorem 3 ensures that, if the RHS evaluates to a value, then the LHS evaluates also to a value and does not raise blame.

\section{RELATED WORK}

Monotonic reference. Siek et al. [2015b] study so-called monotonic references, which are an efficient implementation of mutable references for gradually typed languages. The traditional approach [Herman et al. 2007, 2010] to mutable references in gradual typing is to create a proxy which performs run-time typechecking on reads and writes. The proxy-based approach works well theoretically, but it incurs significant run-time overhead even for statically typed parts in a gradually typed program. Monotonic references preserve a global invariant that the type of a value in the heap is at least as precise as the types given to references that point to the value. As a consequence, there is no overhead for reading and writing through references of static types, because no type (other than itself) is more precise than a static type. Monotonic references are similar to DTI in the sense that 
monotonic references refine types of values in the heap during evaluation whereas DTI refines type variables to more precise types at run time.

Staged type inference. Shields et al. [1998] propose a combination of dynamic typing and staged computation [Davies and Pfenning 2001; Taha and Sheard 2000] in a statically typed language. In their language, all code values are given a single code type \langle\rangle (without information on the static type of the code as is typical in type systems for staged computation [Davies 1996; Davies and Pfenning 2001; Kim et al. 2006; Taha and Nielsen 2003; Taha and Sheard 2000; Tsukada and Igarashi 2010]) and they are typechecked only when it is executed by eval. Such a "typecheck before eval" strategy is called staged type inference. They also propose an incremental staged type inference, where part of typechecking is performed when code fragments are composed using the quasiquotation mechanism [Bawden 1999; Quine 1981; Steele 1990]; if an obvious inconsistency (such as applying multiplication to a string constant) is found, execution results in a special code constant, which indicates that type checking has failed. This incremental inference is close to ours in that type variables in code fragments are unified and instantiated at run time. However, it is limited for code manipulation. We integrate a similar idea to gradual typing and cast semantics Another (somewhat minor) technical difference is that our semantics does not require full first-order unification at run time: it is always the case that one of the two types to be unified is a type variable and there is no need for occur check.

Type inference for gradually typed languages. Henglein and Rehof [1995] studied a type reconstruction algorithm for a language with the dynamic type, coercions [Henglein 1994], and constraint-based polymorphism. They did discuss the issue of uninstantiated type variables and propose to replace them with the dynamic type before running the program. So, their semantics can be too permissive; it allows successful termination even when there are no static types that can be substituted for uninstantiated type variables without causing run-time errors.

Siek and Vachharajani [2008] propose a type reconstruction algorithm for a gradually typed language and Garcia and Cimini [2015] later propose a type inference algorithm for a very similar language ${ }^{9}$ ITGL with a principal type property. The key idea of Garcia and Cimini is to infer only static types for type variables, which represent omitted type annotations. They have also discussed how the surface language where type annotations are inferred can be translated to a blame calculus, where types are explicit not only in lambda abstractions but also in casts, by showing type-directed translation into a slight variant ${ }^{10}$ of the Polymorphic Blame Calculus [Ahmed et al. 2011, 2017]. As we have discussed, this translation raises two problems: (1) The interpretation of type variables left undecided by type inference is not clear-if they are interpreted as fresh base types, a program may fail earlier at run time than a type-substitution instance of it; and (2) the semantics based on the Polymorphic Blame Calculus does not match the intuition that let $x=w$ in $f$ behaves the same as $f[x:=w]$, which we believe is desirable especially in languages where type abstractions and applications are implicit. Actually, Garcia and Cimini mention that the semantics of the ITGL can be defined by first expanding let and translating into a simply typed blame calculus-although it would still have the first problem-but differences of the two translations are not discussed.

Rastogi et al. [2012] present a flow-based type inference algorithm for ActionScript. The type system is based on subtyping rather than polymorphism. Their type inference algorithm assigns a type without type variables for all type variables, so the problem we have tackled in this paper does not arise.

\footnotetext{
${ }^{9}$ In fact, their type systems are shown to be equivalent in a certain sense [Garcia and Cimini 2015]. The algorithm in Siek and Vachharajani [2008] replaces uninstantiated type variables with the dynamic type, similarly to Henglein [1994].

${ }^{10}$ Type variables, which are bound by type abstraction, and type parameters, which correspond to type variables left undecided and occur free in a program, are distinguished in the syntax.
} 
More recently, Xie et al. [2018] introduce the dynamic type into the Odersky-Läufer type system [Odersky and Läufer 1996] for higher-rank polymorphism. They also develop a bidirectional algorithmic type system, which is used to infer static monotypes for missing type declarations at lambda abstractions and type instantiations for polymorphic types. As we have already discussed, they point out that the interpretation of type variables left undecided by type inference affects the run-time behavior of a program and suggest to substitute the dynamic type for such undecided type variables, which is basically the same idea as Henglein [1994] and Siek and Vachharajani [2008], although some refinement based on static and gradual type parameters [Garcia and Cimini 2015] is discussed.

Polymorhic gradual typing. Ahmed et al. [2011, 2017] propose the Polymorphic Blame Calculus, which is based on System F [Girard 1972; Reynolds 1974]. They introduce the notion of type bindings to enforce parametricity. Igarashi et al. [2017] also propose a polymorphic gradually typed language and a similar polymorphic blame calculus. As we have already discussed above, it has an undesirable consequence to implement polymorphic let via type abstraction and type application with dynamic enforcement of parametricity.

Nongeneralizable type variables in OCaml. In the implementation ocaml, a read-eval-print loop for OCaml, when the type of a given expression (bound by a top-level let) contains type variables which cannot be generalized due to (relaxed) value restriction [Garrigue 2004; Wright 1995], the expression is accepted with those type variables being left uninstantiated. ${ }^{11}$ (In Standard ML, such an input requires a type annotation; otherwise it will be rejected.) The following session with ocaml (taken from the OCaml FAQ) shows an example:

\# let $r=$ ref []$;$;

val $r$ : ' _weak1 list ref $=\{$ contents $=[]\}$

Here, '_weak1 stands for a type variable which was undecided by type inference but cannot be used polymorphically. Such type variables will be instantiated as the declared variable is used in a more specific context. For example, the following input enforces that the contents of $r$ have to be an integer list:

\# $r:=42:: \quad ! r ;$

- : unit $=()$

Now, the type of $r$ changes to int list ref by substituting int for '_weak1:

$\# \mathrm{r} ;$;

- : int list ref $=\{$ contents $=[42]\}$

This behavior is similar to our DTI in the sense that type variables left undecided by type inference are instantiated according to their use. However, instantiation of type variables is caused by (compile-time) type inference, which takes place before evaluating every input expression.

\section{CONCLUSION}

We have developed a new blame calculus $\lambda_{B}^{\text {DTI }}$ with DTI, which infers types for undecided (at compiletime) type variables along evaluation. We have also extended $\lambda_{\mathrm{B}}^{\mathrm{DTI}}$ to let-polymorphism and proposed a nonparametric semantics, which we argue is better than the PBC-based approach [Ahmed et al. 2011, 2017] for languages with implicit type abstraction and application. Our calculus can be used as an intermediate language to give semantics to the ITGL by Garcia and Cimini [2015]. We have shown the type safety of $\lambda_{\mathrm{B}}^{\mathrm{DTI}}$ and the soundness and completeness of DTI. We have also shown the type safety and the gradual guarantee in the ITGL. To our knowledge, the gradual guarantee

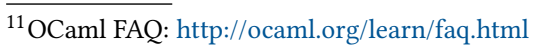


for a gradually typed language with let-polymorphism is shown for the first time. Although we leave a more formal investigation for future work, we have pointed out a relationship between the gradual guarantee and the soundness-completeness property of DTI.

We have implemented a prototype evaluator of DTI, which is used in an interpreter of the ITGL but leave the study of efficiency issues on DTI to future work. We are interested in the impact of run-time overhead incurred by DTI and an efficient implementation to address it. Note that not all type variables are subject to dynamic type inference and some of the run-time type information can be erased by introducing the distinction between static and gradual type variables [Garcia and Cimini 2015; Igarashi et al. 2017]. In particular, if a program does not use the dynamic type $\star$ at all, we expect that a program can be run without passing type information or instantiating type variables dynamically. Another direction for efficiency improvement is to integrate DTI with space-efficient cast calculi [Herman et al. 2007, 2010; Siek and Wadler 2010]. The space-efficient calculi make efficient use of the memory space for casts generated at run time by "merging" two casts into one dynamically. It is interesting to investigate how to merge casts referring to type variables effectively.

We expect DTI can be extended to other typing features such as subtyping. An obvious candidate to apply DTI would be the gradual extension of higher-rank polymorphism [Xie et al. 2018], which we also leave for future work.

\section{ACKNOWLEDGMENTS}

We would like to thank anonymous reviewers from both PC and AEC for valuable comments and Yuu Igarashi for the fruitful discussions. This work was supported in part by the JSPS KAKENHI Grant Number JP17H01723 (Igarashi) and ERATO HASUO Metamathematics for Systems Design Project (No. JPMJER1603), JST (Sekiyama).

\section{REFERENCES}

Martín Abadi, Luca Cardelli, Benjamin C. Pierce, and Gordon D. Plotkin. 1991. Dynamic Typing in a Statically Typed Language. ACM Transactions on Programming Languages and Systems 13, 2 (1991), 237-268. https://doi.org/10.1145/103135.103138

Amal Ahmed, Robert Bruce Findler, Jeremy G. Siek, and Philip Wadler. 2011. Blame for all. In Proc. of ACM POPL. 201-214. https://doi.org/10.1145/1926385.1926409

Amal Ahmed, Dustin Jamner, Jeremy G. Siek, and Philip Wadler. 2017. Theorems for free for free: parametricity, with and without types. PACMPL 1, ICFP (2017), 39:1-39:28. https://doi.org/10.1145/3110283

Felipe Bañados Schwerter, Ronald Garcia, and Éric Tanter. 2014. A theory of gradual effect systems. In Proc. of ACM ICFP. 283-295. https://doi.org/10.1145/2628136.2628149

Felipe Bañados Schwerter, Ronald Garcia, and Éric Tanter. 2016. Gradual type-and-effect systems. Fournal of Functional Programming 26 (2016), e19. https://doi.org/10.1017/S0956796816000162

Alan Bawden. 1999. Quasiquotation in Lisp. In Proc. of ACM PEPM. 4-12.

Nikolaj Skallerud Bjørner. 1994. Minimal Typing Derivations. In Proceedings of the ACM SIGPLAN Workshop on ML and its Applications. 120-126.

Gilad Bracha and David Griswold. 1993. Strongtalk: Typechecking Smalltalk in a Production Environment. In Proc. of ACM OOPSLA. 215-230. https://doi.org/10.1145/165854.165893

Val Breazu-Tannen, Thierry Coquand, Carl A. Gunter, and Andre Scedrov. 1991. Inheritance as Implicit Coercion. Inf. Comput. 93, 1 (1991), 172-221. https://doi.org/10.1016/0890-5401(91)90055-7 Also in Carl A. Gunter and John C. Mitchell, editors, Theoretical Aspects of Object-Oriented Programming: Types, Semantics, and Language Design (MIT Press, 1994.

Robert Cartwright and Mike Fagan. 1991. Soft Typing. In Proc. of ACM PLDI. 278-292. https://doi.org/10.1145/113445.113469

Matteo Cimini and Jeremy G. Siek. 2016. The Gradualizer: A Methodology and Algorithm for Generating Gradual Type Systems. In Proc. of ACM POPL. 443-455. https://doi.org/10.1145/2837614.2837632

Matteo Cimini and Jeremy G. Siek. 2017. Automatically generating the dynamic semantics of gradually typed languages. In Proc. of ACM POPL. 789-803. http://dl.acm.org/citation.cfm?id=3009863

Rowan Davies. 1996. A Temporal-Logic Approach to Binding-Time Analysis. In Proc. of IEEE LICS. 184-195.

Rowan Davies and Frank Pfenning. 2001. A Modal Analysis of Staged Computation. J. ACM 48, 3 (2001), 555-604. 
Robert Bruce Findler and Matthias Felleisen. 2002. Contracts for higher-order functions. In Proc. of ACM ICFP. 48-59. https://doi.org/10.1145/581478.581484

Cormac Flanagan and Matthias Felleisen. 1999. Componential Set-Based Analysis. ACM Transactions on Programming Languages and Systems 21, 2 (1999), 370-416. https://doi.org/10.1145/316686.316703

Ronald Garcia and Matteo Cimini. 2015. Principal Type Schemes for Gradual Programs. In Proc. of ACM POPL. 303-315. https://doi.org/10.1145/2676726.2676992

Ronald Garcia, Alison M. Clark, and Éric Tanter. 2016. Abstracting gradual typing. In Proc. of ACM POPL. 429-442. https://doi.org/10.1145/2837614.2837670

Jacques Garrigue. 2004. Relaxing the Value Restriction. In Proc. of FLOPS (LNCS), Vol. 2998. Springer, 196-213. https: //doi.org/10.1007/978-3-540-24754-8_15

Jean-Yves Girard. 1972. Interprétation fonctionnelle et élimination des coupures de l'arithmétique d'ordre supérieur. Thèse d'état. Université Paris VII. Summary in Proc. of the Second Scandinavian Logic Symposium, 1971 (63-92).

Robert Harper and John C. Mitchell. 1993. On The Type structure of Standard ML. ACM Transactions on Programming Languages and Systems 15, 2 (1993), 211-252.

Fritz Henglein. 1994. Dynamic Typing: Syntax and Proof Theory. Sci. Comput. Program. 22, 3 (1994), 197-230. https: //doi.org/10.1016/0167-6423(94)00004-2

Fritz Henglein and Jakob Rehof. 1995. Safe polymorphic type inference for a Dynamically Typed Language: Translating Scheme to ML. In ACM Conference on Functional Programming and Computer Architecture. 192-203.

David Herman, Aaron Tomb, and Cormac Flanagan. 2007. Space-Efficient Gradual Typing. In Proc. of TFP (Trends in Functional Programming), Vol. 8. Intellect, 1-18.

David Herman, Aaron Tomb, and Cormac Flanagan. 2010. Space-efficient gradual typing. Higher-Order and Symbolic Computation 23, 2 (2010), 167-189. https://doi.org/10.1007/s10990-011-9066-z

Yuu Igarashi, Taro Sekiyama, and Atsushi Igarashi. 2017. On polymorphic gradual typing. PACMPL 1, ICFP (2017), 40:1-40:29. https://doi.org/10.1145/3110284

Lintaro Ina and Atsushi Igarashi. 2011. Gradual typing for generics. In Proc. of ACM OOPSLA. 609-624. https://doi.org/10. $1145 / 2048066.2048114$

Ik-Soon Kim, Kwangkeun Yi, and Cristiano Calcagno. 2006. A polymorphic modal type system for Lisp-like multi-staged languages. In Proc. of ACM POPL. 257-268. https://doi.org/10.1145/1111037.1111060

Xavier Leroy. 1993. Polymorphism by Name for References and Continuations. In Conference Record of the Twentieth Annual ACM SIGPLAN-SIGACT Symposium on Principles of Programming Languages, Charleston, South Carolina, USA, January 1993. 220-231. https://doi.org/10.1145/158511.158632

Robin Milner. 1978. A Theory of Type Polymorphism in Programming. f. Comput. System Sci. 17 (1978), 348-375.

Martin Odersky and Konstantin Läufer. 1996. Putting Type Annotations to Work. In Conference Record of POPL'96: The 23rd ACM SIGPLAN-SIGACT Symposium on Principles of Programming Languages, Papers Presented at the Symposium, St. Petersburg Beach, Florida, USA, January 21-24, 1996. 54-67. https://doi.org/10.1145/237721.237729

Willard Van Orman Quine. 1981. Mathematical Logic (revised edition ed.). Harvard University Press.

Aseem Rastogi, Avik Chaudhuri, and Basil Hosmer. 2012. The ins and outs of gradual type inference. In Proc. of ACM POPL. 481-494. https://doi.org/10.1145/2103656.2103714

John Reynolds. 1974. Towards a Theory of Type Structure. In Proc. Colloque sur la Programmation (LNCS), Vol. 19. Springer, 408-425.

John C. Reynolds. 1983. Types, Abstraction and Parametric Polymorphism. In IFIP Congress. 513-523.

Ilya Sergey and Dave Clarke. 2012. Gradual Ownership Types. In Proc. of ESOP (LNCS), Vol. 7211. Springer, 579-599. https://doi.org/10.1007/978-3-642-28869-2_29

Mark Shields, Tim Sheard, and Simon L. Peyton Jones. 1998. Dynamic Typing as Staged Type Inference. In Proc. of ACM POPL. 289-302. https://doi.org/10.1145/268946.268970

Jeremy G. Siek and Walid Taha. 2006. Gradual typing for functional languages. In Proc. of Workshop on Scheme and Functional Programming. 81-92.

Jeremy G. Siek and Walid Taha. 2007. Gradual Typing for Objects. In Proc. of ECOOP (LNCS), Vol. 4609. Springer, 2-27. https://doi.org/10.1007/978-3-540-73589-2_2

Jeremy G. Siek and Manish Vachharajani. 2008. Gradual typing with unification-based inference. In Proc. of DLS. 7. https://doi.org/10.1145/1408681.1408688

Jeremy G. Siek, Michael M. Vitousek, Matteo Cimini, and John Tang Boyland. 2015a. Refined Criteria for Gradual Typing. In 1st Summit on Advances in Programming Languages, SNAPL (LIPIcs), Vol. 32. Schloss Dagstuhl - Leibniz-Zentrum für Informatik, 274-293. https://doi.org/10.4230/LIPIcs.SNAPL.2015.274

Jeremy G. Siek, Michael M. Vitousek, Matteo Cimini, Sam Tobin-Hochstadt, and Ronald Garcia. 2015b. Monotonic References for Efficient Gradual Typing. In Proc. of ESOP (LNCS), Vol. 9032. Springer, 432-456. https://doi.org/10.1007/ 978-3-662-46669-8_18

Proc. ACM Program. Lang., Vol. 3, No. POPL, Article 18. Publication date: January 2019. 
Jeremy G. Siek and Philip Wadler. 2010. Threesomes, with and without blame. In Proc. of ACM POPL. 365-376. https: //doi.org/10.1145/1706299.1706342

Guy L. Steele, Jr. 1990. Common LISP: the language, 2nd Edition. Digital Pr. http://www.worldcat.org/oclc/20631879

Walid Taha and Michael Florentin Nielsen. 2003. Environment classifiers. In Proc. of ACM POPL. 26-37. https://doi.org/10. 1145/640128.604134

Walid Taha and Tim Sheard. 2000. MetaML and multi-stage programming with explicit annotations. Theoretical Computer Science 248, 1-2 (2000), 211-242. https://doi.org/10.1016/S0304-3975(00)00053-0

Satish R. Thatte. 1990. Quasi-Static Typing. In Proc. of ACM POPL. 367-381. https://doi.org/10.1145/96709.96747

Sam Tobin-Hochstadt and Matthias Felleisen. 2008. The design and implementation of typed Scheme. In Proc. of ACM POPL. 395-406. https://doi.org/10.1145/1328438.1328486

Takeshi Tsukada and Atsushi Igarashi. 2010. A Logical Foundation for Environment Classifiers. Logical Methods in Computer Science 6, 4:8 (2010), 1-43. https://doi.org/10.2168/LMCS-6(4:8)2010

Philip Wadler and Robert Bruce Findler. 2009. Well-Typed Programs Can't Be Blamed. In Proc. of ESOP (LNCS), Vol. 5502. Springer, 1-16. https://doi.org/10.1007/978-3-642-00590-9_1

Andrew K. Wright. 1995. Simple Imperative Polymorphism. Lisp and Symbolic Computation 8, 4 (1995), 343-355.

Andrew K. Wright and Matthias Felleisen. 1994. A Syntactic Approach to Type Soundness. Information and Computation 115, 1 (1994), 38-94.

Ningning Xie, Xuan Bi, and Bruno C. d. S. Oliveira. 2018. Consistent Subtyping for All. In Programming Languages and Systems - 27th European Symposium on Programming, ESOP 2018, Held as Part of the European foint Conferences on Theory and Practice of Software, ETAPS 2018, Thessaloniki, Greece, April 14-20, 2018, Proceedings. 3-30. https://doi.org/10.1007/ 978-3-319-89884-1_1 